\title{
Complex microstructure and magnetism in polymorphic CaFeSeO
}

Simon J. Cassidy, ${ }^{1,2}$ Maria Batuk, ${ }^{3}$ Dmitry Batuk, ${ }^{3}$ Joke Hadermann, ${ }^{3}$ Daniel N.

Woodruff, ${ }^{1}$ Amber L. Thompson, ${ }^{1}$ and Simon J. Clarke ${ }^{1 *}$

${ }^{1}$ Department of Chemistry, University of Oxford, Inorganic Chemistry Laboratory, South Parks Road, Oxford, OX1 3QR, UK. ²Diamond Light Source Ltd., Harwell Science and Innovation Campus, Didcot, OX11 ODE, UK. ${ }^{3}$ Electron Microscopy for Materials Science (EMAT), University of Antwerp, Groenenborgerlaan 171, B-2020 Antwerp, Belgium.

simon.clarke@chem.ox.ac.uk 


\section{Abstract}

The structural complexity of the antiferromagnetic oxide selenide $\mathrm{CaFeSeO}$ is described. The compound contains puckered $\mathrm{FeSeO}$ layers composed of $\mathrm{FeSe}_{2} \mathrm{O}_{2}$ tetrahedra sharing all their vertexes. Two polymorphs coexist that can be derived from an archetype BaZnSO structure by cooperative tilting of the $\mathrm{FeSe}_{2} \mathrm{O}_{2}$ tetrahedra. The polymorphs differ in the relative arrangement of the puckered layers of vertex-linked $\mathrm{FeSe}_{2} \mathrm{O}_{2}$ tetrahedra. In a non-centrosymmetric $\mathrm{Cmc}_{1}$ polymorph ( $a=3.89684(2) \AA, b=13.22054(8) \AA, c=5.93625(2) \AA$ ) the layers are related by the $C$ centring translation while in a centrosymmetric Pmon polymorph, with a similar cell metric ( $a$ $=3.89557(6) \AA, b=13.2237(6) \AA, c=5.9363(3) \AA$ ) , the layers are related by inversion. The compound shows long range antiferromagnetic order below a Neél temperature of 159(1) K with both polymorphs showing antiferromagnetic coupling via Fe-O-Fe linkages and ferromagnetic coupling via Fe-Se-Fe linkages within the FeSeO layers. The magnetic susceptibility also shows evidence for weak ferromagnetism which is modeled in the refinements of the magnetic structure as arising from an uncompensated spin canting in the non-centrosymmetric polymorph. There is also a spin glass component to the magnetism which likely arises from the disordered regions of the structure evident in the transmission electron microscopy. 


\section{Introduction}

Oxide chalcogenides are a relatively unexplored series of compounds. The different sizes and chemistry of the heavier chalcogenide $(C h)$ ions $\mathrm{S}^{2-}, \mathrm{Se}^{2-}$ and $\mathrm{Te}^{2-}$ compared with oxide mean that anion ordering is the norm in oxide chalcogenides. In multinary compounds, partitioning of metal cations may accompany this ordering, with the cations of less electropositive elements being coordinated by the heavier chalcogenide and the cations of the more electropositive elements being coordinated by oxide. This often leads to layered crystal structures. ${ }^{1}$ Furthermore, lower oxidation states of transition metals are stabilised in oxide chalcogenides than is the case in pure oxides. For example the layered compounds LaCuChO, which have been investigated as possible p-type transparent conductors under hole doping, ${ }^{2}$ contain LaO layers with oxide in tetrahedral coordination by $\mathrm{La}$, and $\mathrm{CuCh}$ layers with the highly chalcophilic $\mathrm{Cu}^{+}$ions in tetrahedral coordination by $\mathrm{Ch}^{2-}$. In this case hole doping leads to partial oxidation of the heavier chalcogenide ion, rather than pure oxidation of $\mathrm{Cu}^{+}$, as would occur in oxides. Hence in LaCuChO compounds hole doping leads to depletion of anion-based states at the top of the $\mathrm{Cu}-3 d / C h-n p$ valence band. The importance of pursuing the multianion theme is underlined by the discovery that electron-doping of the layered compound $\mathrm{LaFeAsO}$, isostructural with $\mathrm{LaCuChO}$, to make $\mathrm{LaFeAsO}_{1-x} \mathrm{~F}_{x}{ }^{3}$ resulted in a new series of high temperature superconductors containing either iron arsenide or iron selenide layers in which $\mathrm{FeAs}_{4}$ or $\mathrm{FeSe}_{4}$ tetrahedra share edges, resulting in close approach of $\mathrm{Fe}^{2+}$ ions and placing the compounds in the metallic, rather than Mott-Hubbard insulating regime. This discovery has led to numerous investigations of new iron pnictides and chalcogenides, and the observation that high temperature superconductivity is rather ubiquitous in compounds with such FeAs and FeSe layers and with the appropriate electron count. An extensive review of this area is provided by Hosono et al. ${ }^{4}$ including a description of the attempted synthesis of many compositions which did not result in single phase products.

Here we report on the synthesis and characterisation of the orange-colored antiferromagnet $\mathrm{CaFeSeO}$. The work was motivated by the prospects of discovering further compounds with iron in the +2 oxidation state containing FeSe layers with edge-sharing $\mathrm{FeSe}_{4}$ tetrahedra which might be potential superconductors. However, it was also carried out in the knowledge that in $\mathrm{BaZnSO}^{5}$ and $\mathrm{BaCoSO}{ }^{6}$ the cations of the less electropositive metals are in $\mathrm{MS}_{2} \mathrm{O}_{2}$ tetrahedra which share all their vertexes, and in $\mathrm{CaZnSO}^{7} \mathrm{CaCoSO}^{8}$ and $\mathrm{CaFeSO}^{9,10}$ the $\mathrm{Zn}^{2+}, \mathrm{Co}^{2+}$ or $\mathrm{Fe}^{2+}$ ions are in $\mathrm{MS}_{3} \mathrm{O}$ tetrahedra which share all their sulfide vertexes. The vertex sharing leads to increased $M-M$ distances and hence localised electron behaviour in $\mathrm{BaCOSO}$ and $\mathrm{CaFeSO}$, rather than the metallic behaviour in the iron-based superconductors. 
Attempts to synthesise $\mathrm{CaFeSeO}$ are listed as unsuccessful in the recent review of new materials related to iron-based superconductors. ${ }^{4}$ We show that it requires a very narrow temperature window for synthesis. Han et al. ${ }^{11}$ have described semiconducting CaFeSeO with a $1.8 \mathrm{eV}$ band gap from single crystal diffraction investigations on material extracted from a multi-phase product. We show, using high resolution powder diffraction and high resolution transmission electron microscopy (TEM) that stoichiometric samples of $\mathrm{CaFeSeO}$ consist of two polymorphs, one of which is the form reported by Han et al., ${ }^{11}$ and that the two polymorphs correspond to two different distortions of the $\mathrm{BaZnSO}$ structure, with different relative arrangements of the $\mathrm{FeSe}_{2} \mathrm{O}_{2}$ tetrahedra.

\section{Experimental Methods}

Synthesis. CaFeSeO was synthesised by reaction of $\mathrm{CaO}$ (Sigma Aldrich $\geq 99.99 \%$ ) and FeSe in a 1:1 ratio. The FeSe precursor was formed by reaction of iron (Alfa Aesar $99.998 \%$ ) and selenium (Alfa Aesar $99.999 \%)$ powders as described previously. ${ }^{12}$ Direct reaction of $\mathrm{CaO}$ with iron and selenium powders, without the prior synthesis of iron selenide was found to produce the same product with a higher level of impurities. $\mathrm{CaO}$ and FeSe were ground together inside an argon-filled dry glovebox using an agate pestle and mortar. The ground powder was pressed into a pellet, placed inside an alumina crucible, and sealed inside an evacuated silica ampoule. Synthesis was attempted using a range of conditions of temperature and reaction duration, but it was found that a narrow temperature range between $740^{\circ} \mathrm{C}$ and $750^{\circ} \mathrm{C}$ was required to avoid substantial proportions of impurity phases. Three samples of $\mathrm{CaFeSeO}$ prepared in this temperature range are considered in this work. Samples 1 and 2 were each heated in ampoules to $740{ }^{\circ} \mathrm{C}$ at $5^{\circ} \mathrm{C} \mathrm{min}^{-1}$, the temperature was maintained for 60 hours, then the furnace was switched off and the sample allowed to cool inside the furnace ("furnace cooling"). The same synthesis procedure was employed for sample 3, with an annealing temperature of $750{ }^{\circ} \mathrm{C}$. After cooling, the ampoule was returned to the argonfilled glovebox and the pellet was ground to a powder of orange-brown color. CaFeSeO was found to be stable under air as judged by powder X-ray diffraction, however samples were stored in an argonfilled, dry glovebox to avoid possible long-term degradation and many of the reported measurements have been performed under anaerobic conditions. A small CaSe impurity was accounted for in the structure refinements against powder diffraction data. Repeated annealing, and in one case a 28 day annealing of sample 2 at $740{ }^{\circ} \mathrm{C}$, followed by furnace cooling, were found not to improve the phase purity. 
Structural Characterization. Powder X-ray Diffraction (PXRD) measurements for monitoring the course of the reactions and for preliminary structure solution and refinement were performed using a PANalytical X'Pert PRO instrument equipped with a Ge(111) monochromator to select $\mathrm{Cu}-\mathrm{K} \alpha_{1}$ radiation. PXRD measurements for detailed structural characterisation between 100 and $300 \mathrm{~K}$ used beam line $I 11$ at the Diamond Light Source, UK ${ }^{13}$ with $0.82628 \AA$ X-rays. The Multi-Analyser Crystal (MAC) detector and the MYTHEN position sensitive detector (PSD) (for extremely high signal to noise patterns) were used. For variable temperature measurements a PXRD pattern was collected every 24 seconds using the PSD detector as the sample was cooled using a Cryostream from $295 \mathrm{~K}$ to $110 \mathrm{~K}$ at a rate of $6 \mathrm{~K} \mathrm{~min}^{-1}$. Powder Neutron Diffraction (PND) measurements used the WISH, GEM and POLARIS diffractometers at the ISIS Facility, UK. WISH is optimised for $d$-spacings exceeding $1 \AA$ and was used for the low temperature characterisation of the crystal and magnetic structure of Sample 1. GEM and POLARIS reach shorter $d$-spacings reducing correlation between structural parameters and were used for the ambient temperature structural characterisation of Sample 3. Single Crystal Xray diffraction (SCXRD) was carried out on small $(\sim 5 \times 5 \times 2 \mu \mathrm{m})$ crystals from Sample 1 using beam line $119^{14}$ at Diamond with $0.68890 \AA$ X-rays. Structure solution using charge flipping, Rietveld refinements of the crystal structure and model-independent Pawley-type fits against powder diffraction data were carried out using Topas Academic Version $5 .{ }^{15} \mathrm{Ab}$ initio structure solution from SCXRD data was performed using SIR92 ${ }^{16}$ implemented within CRYSTALS, ${ }^{17}$ and confirmed the results from the powder measurements, but refinements against these data were hampered by the polymorphism. Electron Diffraction (ED) patterns were acquired with a Philips CM20 transmission electron microscope operated at 200 kV. High-angle annular dark-field scanning transmission electron microscopy (HAADF-STEM) and annular bright-field scanning transmission electron microscopy (ABF-STEM) images, and the ED patterns corresponding to specifically those regions from which the images were obtained, were recorded with a probe aberration corrected FEI Titan ${ }^{3}$ 80-300 microscope at $300 \mathrm{kV}$. TEM samples were prepared by grinding the crushed powder in ethanol and depositing a few drops of the suspension on holey carbon TEM grids. The sample was briefly exposed to air for the SCXRD and TEM experiments but was kept under an argon atmosphere for the PXRD and PND experiments.

Magnetometry. All measurements used a Quantum Design MPMS-XL SQUID magnetometer. The normal state susceptibility was determined by measuring the magnetisation as a function of temperature on warming from 2 to $300 \mathrm{~K}$ after cooling both in a zero applied field: zero-field cooled (ZFC) and in the measuring field: field cooled (FC) of $50 \mathrm{mT}$. Magnetisation isotherms $\left(-5 \leq \mu_{0} H / T\right.$ $\leq 5)$ were measured after cooling the sample from $300 \mathrm{~K}$ to the measurement temperature in a $5 \mathrm{~T}$ 
field. AC magnetometry measurements were carried out at four frequencies in a DC field of $0.3 \mathrm{mT}$ and an oscillating field of amplitude $0.35 \mathrm{mT}$. Samples were sequestered from air in gelatin capsules.

\section{Results and discussion}

\section{Crystal structure determination}

The unit cell of $\mathrm{CaFeSeO}$ was initially indexed from a laboratory powder X-ray diffraction pattern to an orthorhombic unit cell with dimensions of $3.89 \times 13.21 \times 5.92 \AA$. These data were not unequivocal regarding the possible space groups, although $C$-centring was suggested. Initial structural solution was performed using charge flipping, as implemented in Topas Academic, in the space group C222 using high resolution PXRD data from I11. This led to the identification of the $\mathrm{Ca}^{2+}, \mathrm{Fe}^{2+}$, and $\mathrm{Se}^{2-}$ positions in the unit cell. A likely location of the $\mathrm{O}^{2-}$ ion was then determined by placing it at a bonding distance from both $\mathrm{Ca}$ and $\mathrm{Fe}$, whilst avoiding close O-Se contacts. Inspection of the structure revealed additional symmetry elements that suggested $\mathrm{Cmcm}$ as a possible space group. This preliminary structural model in $\mathrm{Cmcm}$ is shown in Figure 1 (d). $\mathrm{Ca}^{2+}, \mathrm{Se}^{2-}$ and $\mathrm{O}^{2-}$ fully occupy 4fold sites in the unit cell, while in this space group iron occupies an 8-fold site with $50 \%$ occupancy. The structure has puckered layers of composition FeSeO extending parallel to the ac plane composed of vertex-linked $\mathrm{FeSe}_{2} \mathrm{O}_{2}$ tetrahedra. These puckered layers are stacked along the $b$ direction and are separated by $\mathrm{Ca}^{2+}$ ions. The modelling of the Fe site as a split site implies the possibility of tetrahedra sharing edges, but this produces unreasonably short Fe-Fe distances so it was assumed that the $\mathrm{FeSe}_{2} \mathrm{O}_{2}$ tetrahedra are joined only through vertexes in these FeSeO puckered layers.

The preliminary structural model deduced in this way can formally be derived from the structure of BaZnSO which is shown in Figure 1(a). The structure of BaZnSO in space group $\mathrm{Cmcm}$ consists of $\mathrm{ZnS}_{2} \mathrm{O}_{2}$ tetrahedra which share all their oxide and sulfide vertices. In this case the $\mathrm{ZnS}_{2} \mathrm{O}_{2}$ tetrahedra are arranged so that the $\mathrm{Zn}-\mathrm{O}-\mathrm{Zn}$ angles are $180^{\circ}$ and the oxide ions are located at an inversion centre ( $4 a$ site in $\mathrm{Cmcm}$ ); the model has all sites fully occupied. In CaFeSeO the incorporation of the smaller $\mathrm{Ca}^{2+}$ cation results in a cooperative tilting of the tetrahedra which reduces the $\mathrm{Fe}-\mathrm{O}-\mathrm{Fe}$ angle greatly from $180^{\circ}$ so that the oxide ion is no longer located on an inversion centre. The cooperative tilting distortions involve anti-phase rotations of neighbouring O-vertex-linked $\mathrm{FeSeO}_{2}$ tetrahedra about the crystallographic $a$ axis (Figure 1). Because of this tilting, retention of the $\mathrm{Cmcm}$ symmetry of BaZnSO requires a split site for the $\mathrm{Fe}$ atom in $\mathrm{CaFeSeO}$ which corresponds to equal representation of the two senses of tilting distortion. The preliminary $\mathrm{Cmcm}$ model with the split Fe 
site produces an unsatisfactory fit (Figure 2(a)) to many of the weak reflections in the synchrotron powder diffraction data.

(a) BaZnOs ( $\mathrm{Cmcm})$

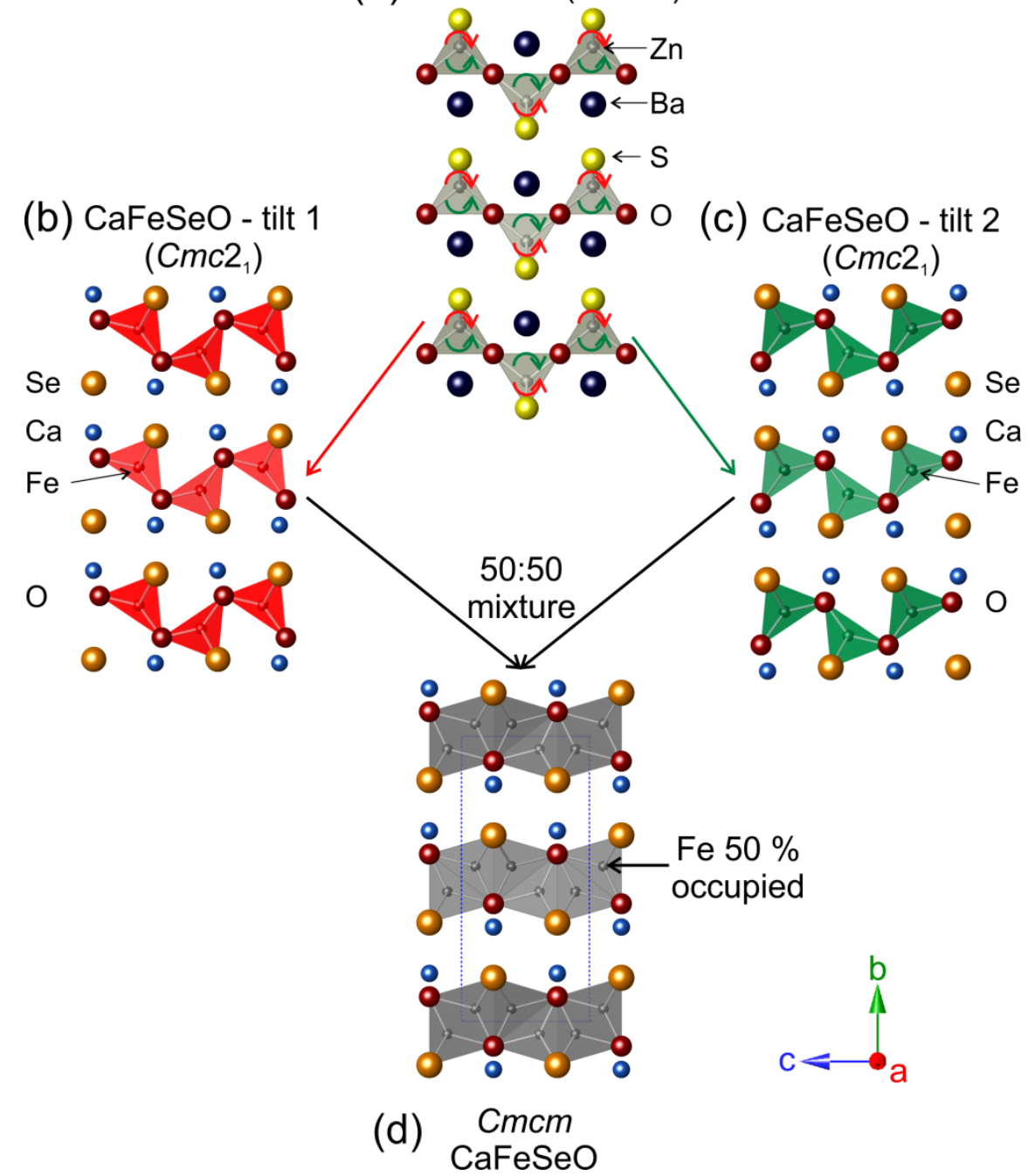

Figure 1. (a) The crystal structure of BaZnSO in $\mathrm{Cmcm}$ shown in projection along the $a$ axis. The red and green arrows define two tilting distortions. (b) and (c) show the effects of applying only one of these distortions to produce (identical) models in $\mathrm{Cmc} 2_{1}$ symmetry which are related by reflection. If these two models are superimposed (d) one retains $\mathrm{Cmcm}$ symmetry, but with a split site for Fe and the requirement that two edge-shared tetrahedra are not both occupied by Fe.

Electron diffraction patterns (Figure 3) confirmed the same $3.89 \times 13.20 \times 5.92 \AA$ orthorhombic unit cell suggested by the PXRD data. The reflection conditions $h k l: h+k=2 n, h 0 l: h, I=2 n, h k 0: h+k=$ $2 n, 0 k l: k=2 n$ correspond to the extinction symbol $C-c-$, allowing space groups $C m c m, C 2 \mathrm{~cm}$, and $\mathrm{Cmc2}_{1}$. A reduction in symmetry to $\mathrm{C2cm}$ allows the relative $x$ coordinates of the sites in the $\mathrm{Cmcm}$ model to be refined, but the need for a split Fe site remains. A model in $C m c 2_{1}$ avoids the need for Fe to occupy a split site, and corresponds to choosing just one of the two senses of tilt distortions of 
the chains of $\mathrm{FeSe}_{2} \mathrm{O}_{2}$ tetrahedra shown in Figure 1 ((b) or (c)) so that the FeSeO layers are related by the $C$ centring translation. The $C 2 \mathrm{~cm}$ model did not produce a significant improvement to the fit, while the model in $\mathrm{Cmc2}_{1}$ did give a much improved Rietveld fit compared with the initial $\mathrm{Cmcm}$ model, as shown in Figure 2(b). However several low intensity reflections that may be indexed on the same orthorhombic unit cell, but which require a lower symmetry than $C m c 2_{1}$, are observable in the synchrotron PXRD data (Figure 2(b)). This indicates that the analysis of the structure as a single $\mathrm{Cmc2}_{1}$ phase gives an incomplete picture of the structure.

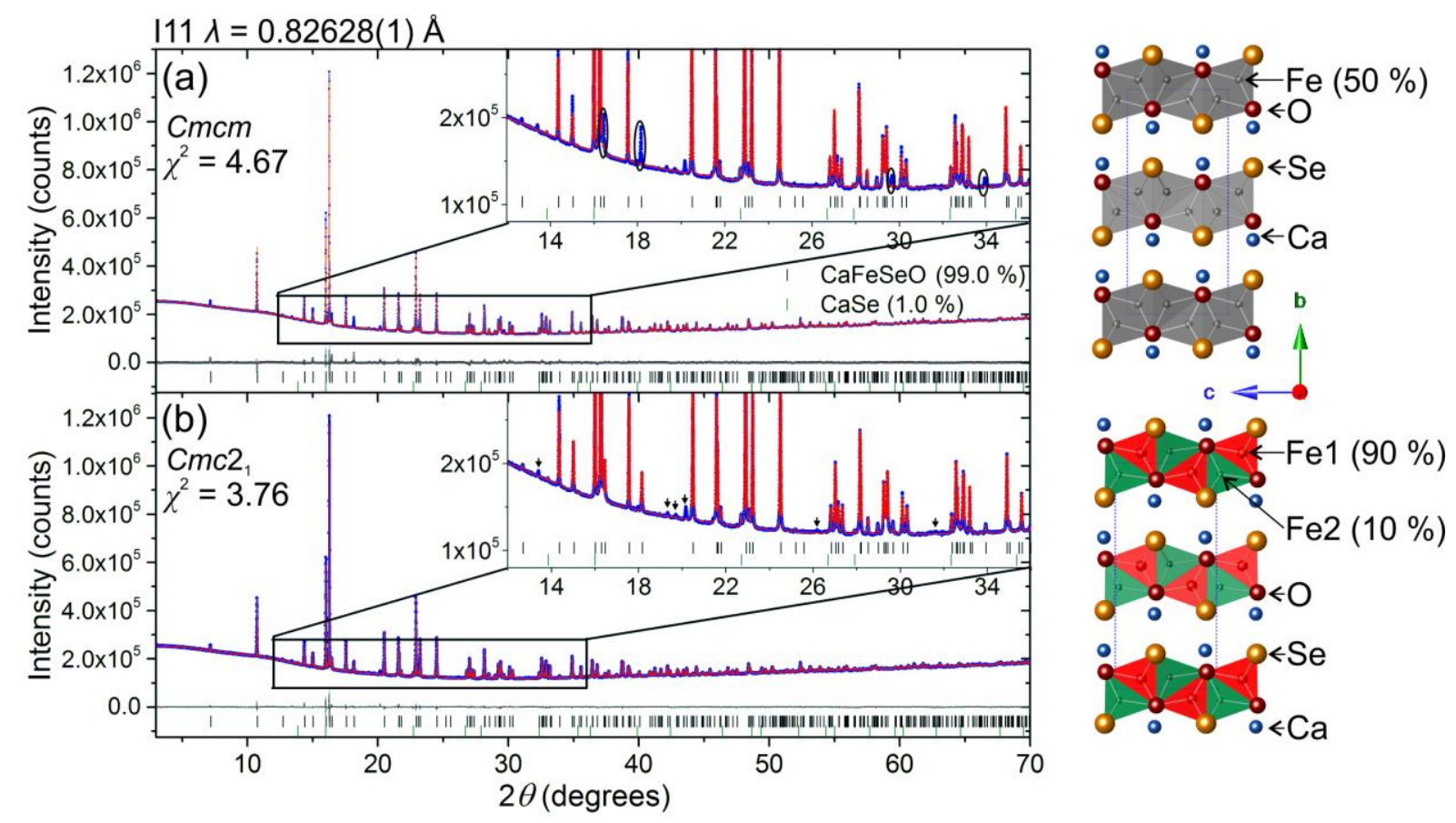

Figure 2 Rietveld refinements against Synchrotron PXRD data of (a) $\mathrm{Cmcm}$ and (b) $\mathrm{Cmc2}{ }_{1}$ models of the $\mathrm{CaFeSeO}$ crystal structure. Data (Blue), fit (red) and difference (grey) are shown. Tick marks indicate reflection positions for $\mathrm{CaFeSeO}$ and $\mathrm{CaSe}$ ( $1 \%$ by mass). Reduction in symmetry $\mathrm{Cmc2}_{1}$ to avoid the Fe ions being split equally between two half-occupied sites significantly improves the intensity match to several predicted peaks circled in the inset of (a), but a few low intensity peaks are left unpredicted as marked by arrows in the inset of (b). Further improvement of the model to account for these reflections is shown later in Figure 5. The apparent 9:1 populations of two iron sites in $\mathrm{Cmc2}_{1}$ is an indication of the inherent phase separation in the system described in the text.

The ED patterns (Figure 3) also showed clear evidence for a structural complexity that is not accounted for by the preliminary models in $\mathrm{Cmcm}$ or $\mathrm{Cmc2}_{1}$ : the [100] and [101] zones contain streaks along the $b^{*}$-axis. On the enlarged fragments of two [101] electron diffraction patterns, shown at the bottom of Figure 3, it can be seen that these streaks consist of low intensity reflections that are very closely spaced. These patterns are taken from different crystals of Sample 1 and show a clear difference in intensity distribution along the "streaks". 

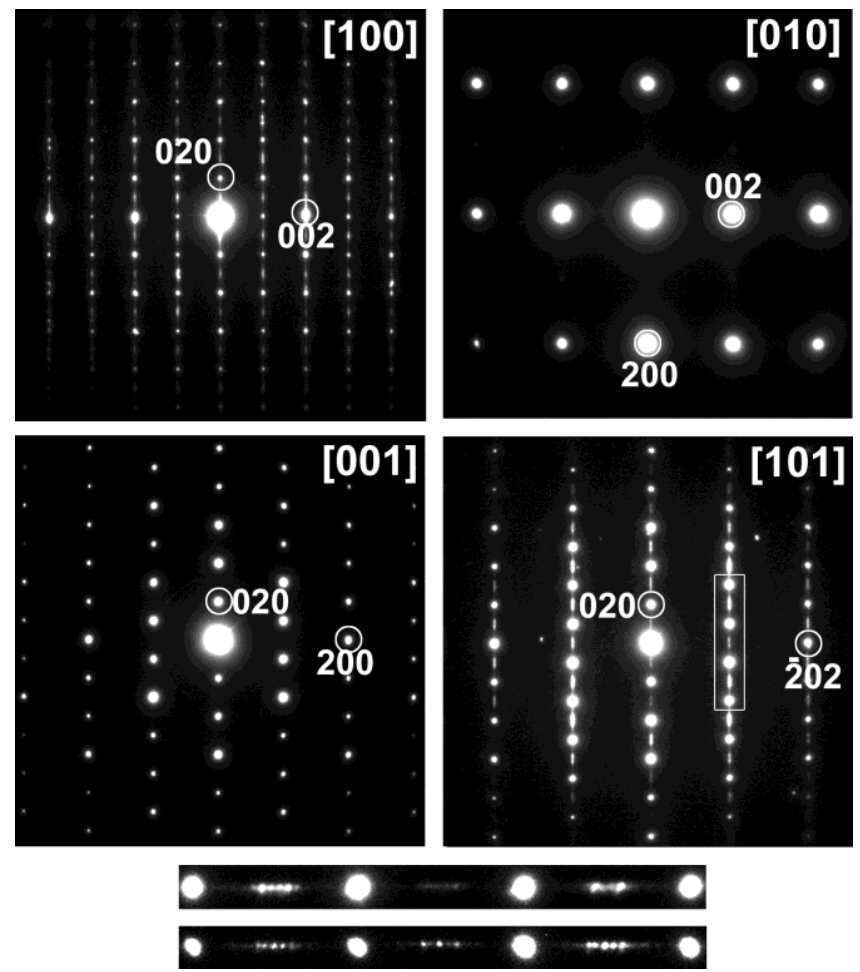

Figure 3. Electron diffraction patterns for $\mathrm{CaFeSeO}$. Some alternative intensity distributions found throughout the sample for the area indicated on the [101] pattern are shown at the bottom of the figure. These enlargements show that the streaks in the [101] zone consist of closely spaced reflections lying between the reflections characteristic of $C$-c- symmetry and that the intensity distribution differs from pattern to pattern.

The HAADF-STEM images and ED patterns collected on smaller crystals (Figure 4) reveal the complex microstructure of $\mathrm{CaFeSeO}$, which accounts for the difficulty of describing the powder diffraction data using a single $C m c 2_{1}$ structure. Two characteristic types of HAADF-STEM images acquired in the sample are shown in Figure 4 (a) and (b). The brightness of the dots on the HAADF-STEM images is proportional to the average atomic number $Z$ of the projected atomic columns, as $Z^{1.6-1.9}$. The brightest dots on the images correspond to the Se columns $\left(Z_{s e}=34\right)$. From the arrangement of the Fe atomic columns $\left(Z_{\mathrm{Fe}}=26\right)$, one can clearly see that two different polymorphs of CaFeSeO exist. In Figure 4(a) a region of the crystal is shown in which FeSeO layers are related to one another by the C-centring translation, giving $C m c 2_{1}$ symmetry, consistent with the initial indexing of the PXRD data and the electron diffraction data. This polymorph corresponds to a distortion of the BaZnSO structure by way of a single sense of tilt by the $\mathrm{FeS}_{2} \mathrm{O}_{2}$ tetrahedra (see Figure 1). In Figure 4(b) a region of the same grain is shown in which the FeSeO layers are related to each other through inversion, giving a unit cell of Pmon (Pnma) symmetry, and a structure that is derived from the 
BaZnSO structure by applying one sense of tilt and then the other to alternate FeSeO layers. This second polymorph is the one that has been described for CaFeSeO by Han et al. ${ }^{11}$ who extracted a single crystal from a multiphase sample. In our samples it appears as a minority phase. This second polymorph has a structure similar to that of $\mathrm{SrZnO}_{2}$. The difference in structure of the two polymorphs can be clearly seen in the corresponding [100] ED patterns, which show different selection rules (Figure 4). 

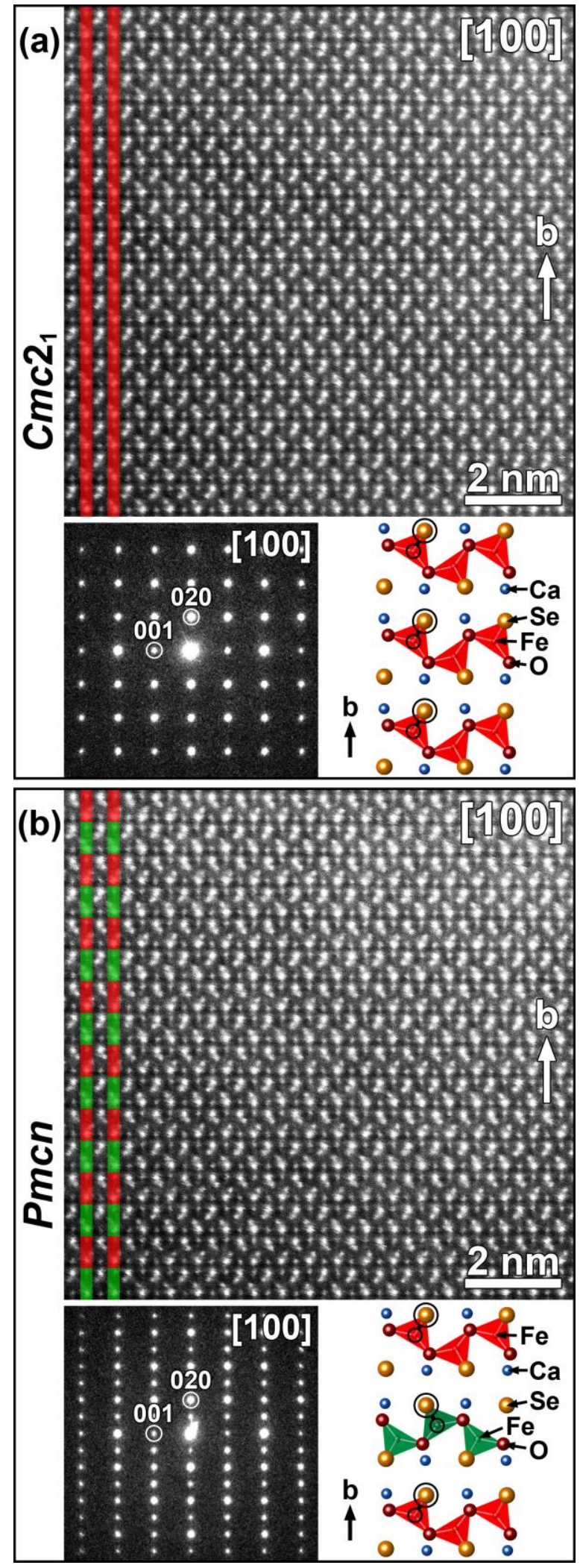

Figure 4 HAADF-STEM images and ED patterns for two polymorphs of CaFeSeO. As shown in the corresponding structural diagrams (and in Figure 1) the $\mathrm{FeSe}_{2} \mathrm{O}_{2}$ tetrahedra can tilt cooperatively about the $a$ axis in two opposite senses (depicted as red and green). The structure with a single sense of the tilts has $\mathrm{Cmc2}{ }_{1}$ symmetry (panel (a)), while the structure with the alternating senses of tilts in the FeSeO blocks stacked along the $b$ axis demonstrates Pmon symmetry (panel (b)). 
Rietveld refinement against a high signal-to-noise PXRD pattern (I11 PSD detector) of sample 1 shown in Figure 5 demonstrates the improved match to the low intensity peaks on inclusion of both $\mathrm{CmC2}_{1}$ and Pmcn phases, without the need to introduce partially occupied sites in a single phase model. Rietveld refinement against PND data (WISH) of sample 1 taken at $180 \mathrm{~K}$ is also shown in Figure 5. High precision structural characterisation was carried out by Rietveld refinements against high resolution PXRD (I11 MAC detector) and PND data extending to the shortest (0.5 $\AA$ ) $d$-spacings (GEM and POLARIS) both taken at ambient temperature on sample 3, which contains slightly less of the Pmon phase, with fits shown in Figure 6 and refined structural parameters given in Tables 1-3. Refined structural parameters for sample 1 are given in Tables S1-S3 in the supporting information. A broad reflection is observed in the WISH neutron diffraction data for sample 1 at $180 \mathrm{~K}$ at a $d$ spacing of $15.2 \AA$ (Figure S2), which may be an indicator of an additional longer range order in the tilts of the tetrahedra in the layers but this could not be indexed to a linear or quadratic expansion of the unit cell. Additional low intensity reflections that do not index to the unit cell of $\mathrm{CaFeSeO}$ are also observed in the high signal-to-noise, PXRD pattern of Sample 1 at $d$-spacings of 4.12, 2.67, 2.64, and $1.50 \AA$, with intensities relative to the strongest peak in the pattern of just $0.4,0.3,0.6$, and 0.2 $\%$, respectively. These peaks may correspond to minuscule fractions of unidentified impurities, but there is evidence from the STEM images of further complexity in the structure that might also be their origin. Attempts to solve the structure using single crystal X-ray diffraction measurements on the 119 diffractometer at Diamond were hampered by the multiphase behaviour within a single crystal which was revealed by the electron diffraction measurements. The $(0 k /)$ simulated precession images from the single crystal X-ray data (Figure 7) show evidence for both phases. 

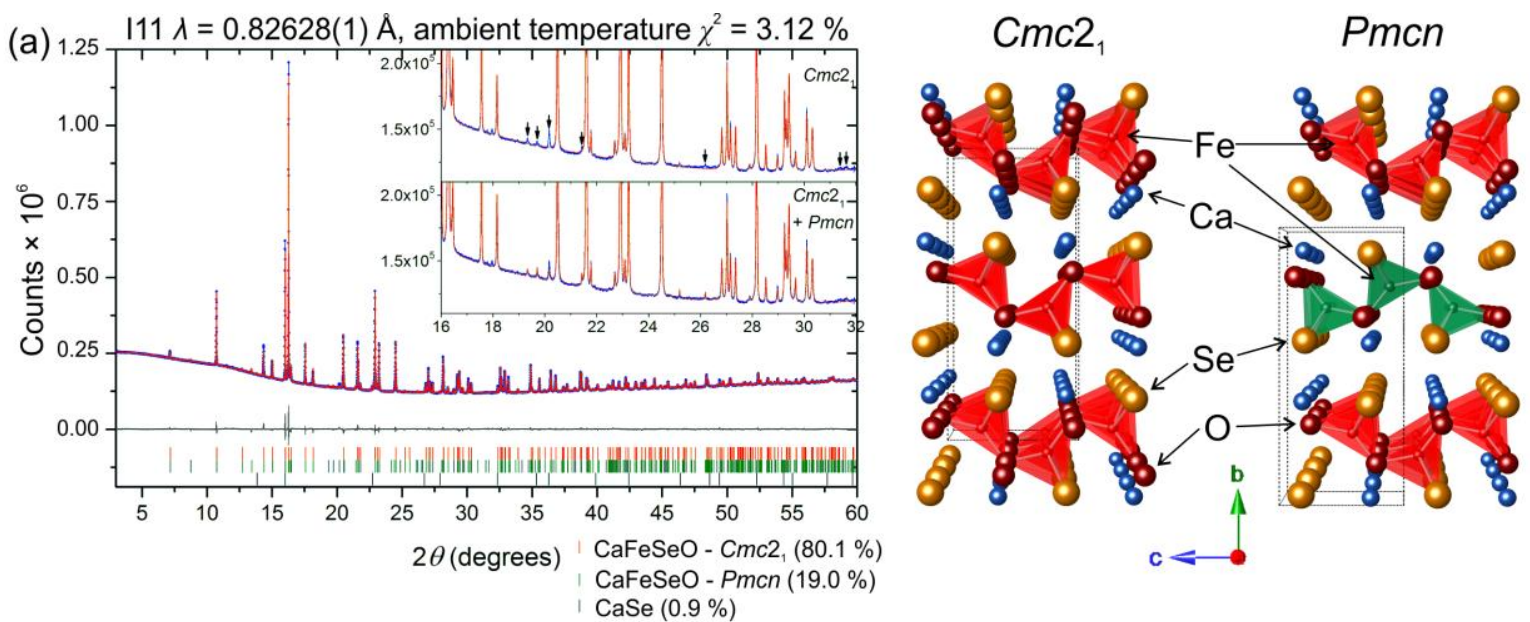

(b)

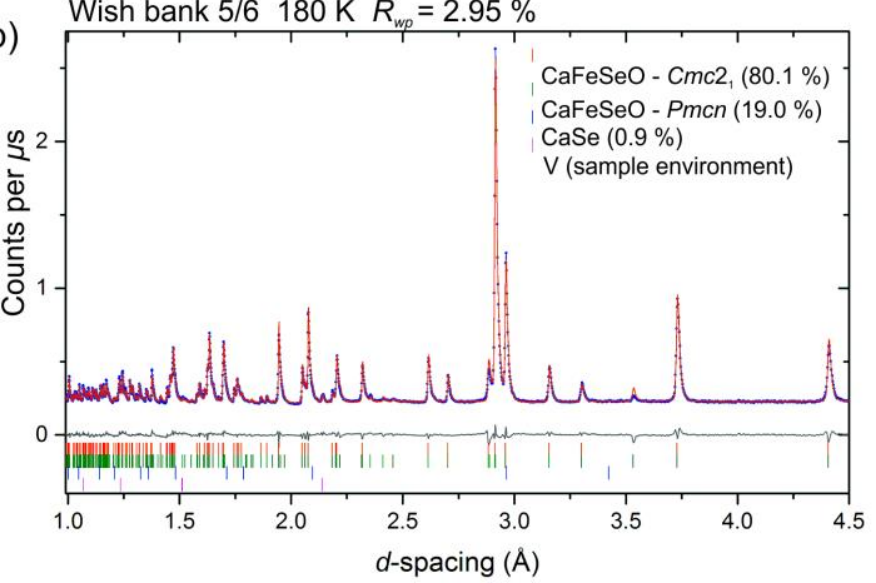

$\mathrm{Cmc2}_{1}$

Pmcn

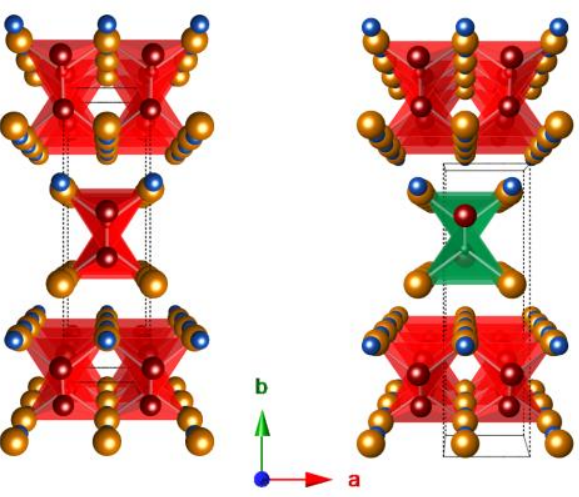

Figure 5 Rietveld refinements against synchrotron PXRD (a) and PND (b) patterns for sample 1 using a combination of the two crystal structure modifications. A comparison with the single phase treatments shown in Figure 2 is given in the inset of (a), showing the improved match to low intensity peaks marked with arrows on inclusion of the Pmcn modification in the model. Data (Blue), fit (red) and difference (grey) are shown. Tick marks indicate reflection positions for the phases present. The fits against the other detector banks of the WISH diffractometer are shown in Figure S2, and refined parameters for (a) and (b) are given in Tables S1-S3. 
(a)

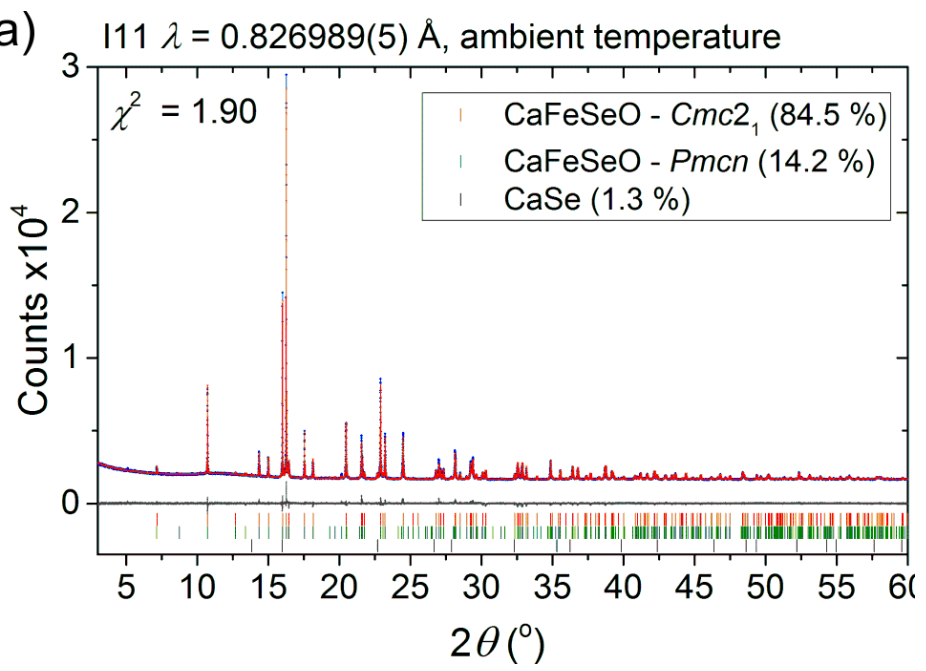

(b) GEM bank 4, ambient temperature

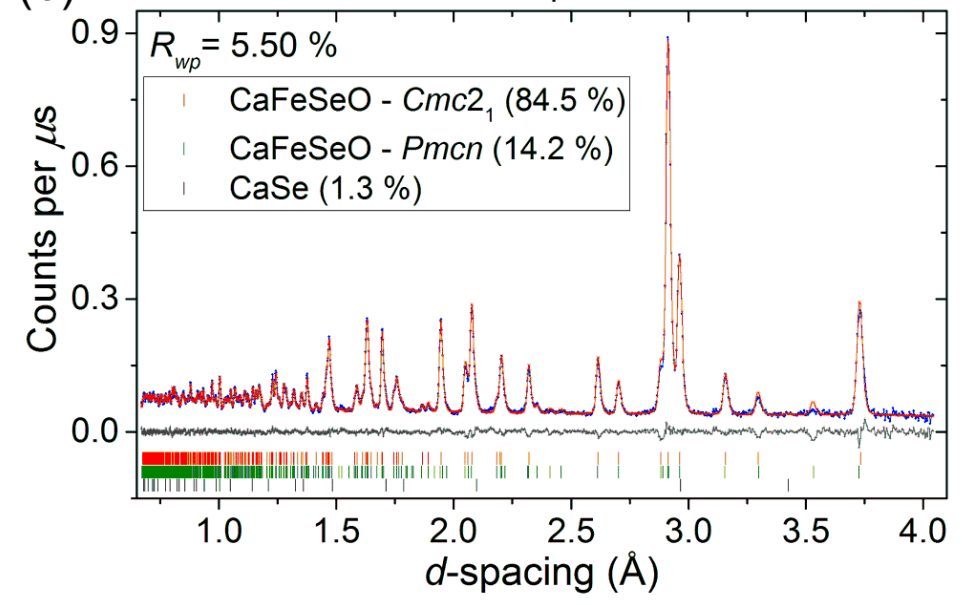

Figure 6 Rietveld refinements using a combination of the two crystal structure modifications against high-resolution synchrotron PXRD (a) and PND (b) patterns for sample 3 collected at ambient temperature. Data (Blue), fit (red) and difference (grey) are shown. Tick marks indicate reflection positions for the phases present. PND refinement was carried out against GEM banks 2-6 and POLARIS banks 4-5 combined, with (b) showing the fit against data from one detector bank. PND was initially carried out using the POLARIS instrument at ISIS, however the lower angle banks 1-3 were unusable due to incorrect sample alignment relative to different portions of the detector bank arrays. The sample was substequently re-measured using the GEM instrument. Fits against the other banks are shown in the supporting information (Figure S3). 
Table 1 Refinement results from a 2 phase fit against PND and PXRD data of CaFeSeO sample 3 at room temperature, corresponding to the fits shown in Figure 6. Results for Sample 1 are given in the supporting information, Tables S1-S3.

\begin{tabular}{|c|c|c|c|c|}
\hline Instrument & \multicolumn{2}{|c|}{ GEM / POLARIS } & \multicolumn{2}{|c|}{ I11 } \\
\hline Temperature & \multicolumn{2}{|c|}{ Room temperature } & \multicolumn{2}{|c|}{ Room temperature } \\
\hline Space group & $\mathrm{Cmc}_{2}(36)$ & $\operatorname{Pmcn}(62)$ & $\mathrm{Cmc2}_{1}(36)$ & $\operatorname{Pmcn}(62)$ \\
\hline$a(\AA)$ & $3.8923(2)^{a}$ & $3.8849(3)$ & $3.89684(2)$ & $3.89557(6)$ \\
\hline$b(\AA)$ & $13.1932(6)$ & $13.200(2)$ & $13.22054(8)$ & $13.2237(6)$ \\
\hline$c(\AA)$ & $5.9263(3)$ & $5.9268(8)$ & $5.93625(2)$ & $5.9363(3)$ \\
\hline$V\left(\AA^{3}\right)$ & $304.33(2)$ & $303.94(6)$ & $305.826(2)$ & $305.80(2)$ \\
\hline$\rho\left(\mathrm{g} \mathrm{cm}^{-3}\right)$ & $4.1662(3)$ & $4.1714(8)$ & $4.14573(4)$ & $4.1461(3)$ \\
\hline $\begin{array}{r}\text { polymorph } \\
\text { fraction }\end{array}$ & $0.86(1)$ & $0.14(1)$ & $0.86(1)$ & $0.14(1)$ \\
\hline $\begin{array}{r}\mathrm{Fe}-\mathrm{O}(\AA ̊) \\
{[1]}\end{array}$ & $\begin{array}{l}1.870(4) \\
1.959(3)\end{array}$ & $\begin{array}{l}1.92(2) \\
2.01(1)\end{array}$ & $\begin{array}{l}1.851(7) \\
2.043(6)\end{array}$ & $\begin{array}{l}1.94(5) \\
2.01(3)\end{array}$ \\
\hline Fe-Se $(\AA ̊)[2]$ & $2.585(2)$ & $2.53(1)$ & $2.581(1)$ & $2.506(8)$ \\
\hline $\mathrm{Ca}-\mathrm{O}(\AA ̊)[2]$ & $2.316(3)$ & $2.32(2)$ & $2.315(4)$ & $2.32(3)$ \\
\hline $\begin{array}{r}\text { Ca-Se }(\AA ̊)[1] \\
{[1]} \\
{[2]}\end{array}$ & $\begin{array}{l}2.943(4) \\
2.996(4) \\
3.044(5)\end{array}$ & $\begin{array}{l}2.94(2) \\
2.99(2) \\
3.05(3)\end{array}$ & $\begin{array}{l}2.922(2) \\
3.028(2) \\
3.034(2)\end{array}$ & $\begin{array}{l}2.96(1) \\
2.99(1) \\
3.07(2)\end{array}$ \\
\hline $\mathrm{Fe}-\mathrm{O}-\mathrm{Fe}\left({ }^{\circ}\right)$ & $111.2(2)$ & 114(1) & $109.2(3)$ & $114(3)$ \\
\hline $\mathrm{O}-\mathrm{Fe}-\mathrm{O}\left({ }^{\circ}\right)$ & $137.7(1)$ & $126.1(7)$ & $134.3(2)$ & $126(1)$ \\
\hline $\begin{array}{r}\mathrm{Fe}-\mathrm{Se}-\mathrm{Fe}\left(\left(^{\circ}\right) /\right. \\
\mathrm{Se}-\mathrm{Fe}-\mathrm{Se}\left({ }^{\circ}\right)\end{array}$ & $97.7(1)$ & $100.5(7)$ & $98.02(5)$ & $102.1(5)$ \\
\hline
\end{tabular}

${ }^{a}$ estimated standard deviations (in parentheses) on refined parameters are those obtained from the refinement software. 
Table 2 structural parameters for sample 3 from Rietveld refinement against PND data using GEM banks 2-6 and POLARIS banks 4 and 5 .

$\mathrm{Cmc2}_{1}$

\begin{tabular}{|l|l|l|l|l|l|l|l|l|}
\hline atom & site & $x$ & $y$ & $z$ & $U_{11}\left(\AA^{2}\right)$ & $U_{22}\left(\AA^{2}\right)$ & $U_{33}\left(\AA^{2}\right)$ & $U_{23}\left(\AA^{2}\right)$ \\
\hline $\mathrm{Ca}$ & $4 a$ & 0 & $0.3313(4)$ & $0.0045(6)$ & $0.002(1)$ & $0.007(1)$ & $0.005(1)$ & $-0.002(1)$ \\
\hline $\mathrm{Fe}$ & $4 a$ & 0 & $0.0414(1)$ & $0.1405(4)$ & $0.0083(8)$ & $0.0155(8)$ & $0.0048(6)$ & $-0.0074(6)$ \\
\hline $\mathrm{Se}$ & $4 a$ & 0 & $0.6539(2)$ & 0 & $0.0055(8)$ & $0.0042(8)$ & $0.0044(8)$ & $0.0016(8)$ \\
\hline $\mathrm{O}$ & $4 a$ & 0 & $0.0755(3)$ & $0.4622(4)$ & $0.006(1)$ & $0.010(2)$ & $0.004(1)$ & $0.004(2)$ \\
\hline
\end{tabular}

Pmcn

\begin{tabular}{|l|l|l|l|l|l|l|l|l|}
\hline atom & site & $x$ & $y$ & $z$ & $U_{11}\left(\AA^{2}\right)^{a}$ & $U_{22}\left(\AA^{2}\right)^{a}$ & $U_{33}\left(\AA^{2}\right)^{a}$ & $U_{23}\left(\AA^{2}\right)^{a}$ \\
\hline $\mathrm{Ca}$ & $4 c$ & 0.25 & $0.584(3)$ & $0.732(2)$ & $0.002(1)$ & $0.007(1)$ & $0.005(1)$ & $-0.002(1)$ \\
\hline $\mathrm{Fe}$ & $4 c$ & 0.25 & $0.1949(9)$ & $0.119(1)$ & $0.0083(8)$ & $0.0155(8)$ & $0.0048(6)$ & $-0.0074(6)$ \\
\hline $\mathrm{Se}$ & $4 c$ & 0.25 & $0.594(1)$ & $0.229(1)$ & $0.0055(8)$ & $0.0042(8)$ & $0.0044(8)$ & $0.0016(8)$ \\
\hline $\mathrm{O}$ & $4 c$ & 0.25 & $0.321(2)$ & $0.281(2)$ & $0.006(1)$ & $0.010(2)$ & $0.004(1)$ & $0.004(2)$ \\
\hline
\end{tabular}

${ }^{a}$ Displacement parameters were constrained to be the same in both phases due to the intrinsic overlap between the peaks from the two phases.

Table 3 structural parameters for sample 3 from Rietveld refinement against I11 (MAC detector) PXRD data.

$\mathrm{Cmc2}_{1}$

\begin{tabular}{|l|l|l|l|l|l|l|l|l|}
\hline atom & site & $x$ & $y$ & $z$ & $U_{11}\left(\AA^{2}\right)$ & $U_{22}\left(\AA^{2}\right)$ & $U_{33}\left(\AA^{2}\right)$ & $U_{23}\left(\AA^{2}\right)$ \\
\hline Ca & $4 a$ & 0 & $0.3303(2)$ & $0.0089(4)$ & $0.008(1)$ & $0.003(2)$ & $0.005(1)$ & $0.000(1)$ \\
\hline Fe & $4 a$ & 0 & $0.0428(1)$ & $0.1400(3)$ & $0.0052(7)$ & $0.0175(8)$ & $0.0064(6)$ & $0.004(1)$ \\
\hline Se & $4 a$ & 0 & $0.65434(8)$ & 0 & $0.0082(5)$ & $0.0062(4)$ & $0.0064(5)$ & $0.0026(5)$ \\
\hline $\mathrm{O}$ & $4 a$ & 0 & $0.0763(5)$ & $0.4759(9)$ & $0.007(1)$ & $0.014(2)$ & $0.009(1)$ & $0.003(2)$ \\
\hline
\end{tabular}

Pmon

\begin{tabular}{|l|l|l|l|l|l|l|l|l|}
\hline atom & site & $x$ & $y$ & $z$ & $U_{11}\left(\AA^{2}\right)^{a}$ & $U_{22}\left(\AA^{2}\right)^{a}$ & $U_{33}\left(\AA^{2}\right)^{a}$ & $U_{23}\left(\AA^{2}\right)^{a}$ \\
\hline $\mathrm{Ca}$ & $4 c$ & 0.25 & $0.583(1)$ & $0.739(2)$ & $0.008(1)$ & $0.003(2)$ & $0.005(1)$ & $0.000(1)$ \\
\hline $\mathrm{Fe}$ & $4 c$ & 0.25 & $0.1948(8)$ & $0.115(2)$ & $0.0052(7)$ & $0.0175(8)$ & $0.0064(6)$ & $0.004(1)$ \\
\hline $\mathrm{Se}$ & $4 c$ & 0.25 & $0.5962(7)$ & $0.236(1)$ & $0.0082(5)$ & $0.0062(4)$ & $0.0064(5)$ & $0.0026(5)$ \\
\hline $\mathrm{O}$ & $4 c$ & 0.25 & $0.322(4)$ & $0.279(5)$ & $0.007(1)$ & $0.014(2)$ & $0.009(1)$ & $0.003(2)$ \\
\hline
\end{tabular}

${ }^{a}$ Displacement parameters were constrained to be the same in both phases due to the intrinsic overlap between the peaks from the two phases.

The ED and SCXRD data indicate that in many crystals the CaFeSeO polymorphs form coherent intergrowths. Apparently this is caused by the extreme similarity of their crystal structures (Figure 5). Both polymorphs consist of similar FeSeO layers with pronounced tilting of the $\mathrm{FeSe}_{2} \mathrm{O}_{2}$ tetrahedra about the $a$ axis. The $\mathrm{Cmc2}_{1}$ polymorph exhibits the same tilting of $\mathrm{FeSe}_{2} \mathrm{O}_{2}$ tetrahedra within every FeSeO layer, whereas the Pmcn polymorph shows alternation of the tilting sense in adjacent layers. Occasional stacking faults in the arrangement of the FeSeO layers result in the smearing of the $h k l$ : $k=2 n+1$ reflections in the ED and SCXRD patterns (Figures 3 and 7, respectively). Local ordered distribution of the stacking faults presumably gives rise in the ED patterns to weak satellite reflection 
arrays centred at the $h k l: k=2 n+1$ positions and running parallel to the $b^{*}$ direction (see enlarged fragments of the ED patterns in Figure 3).

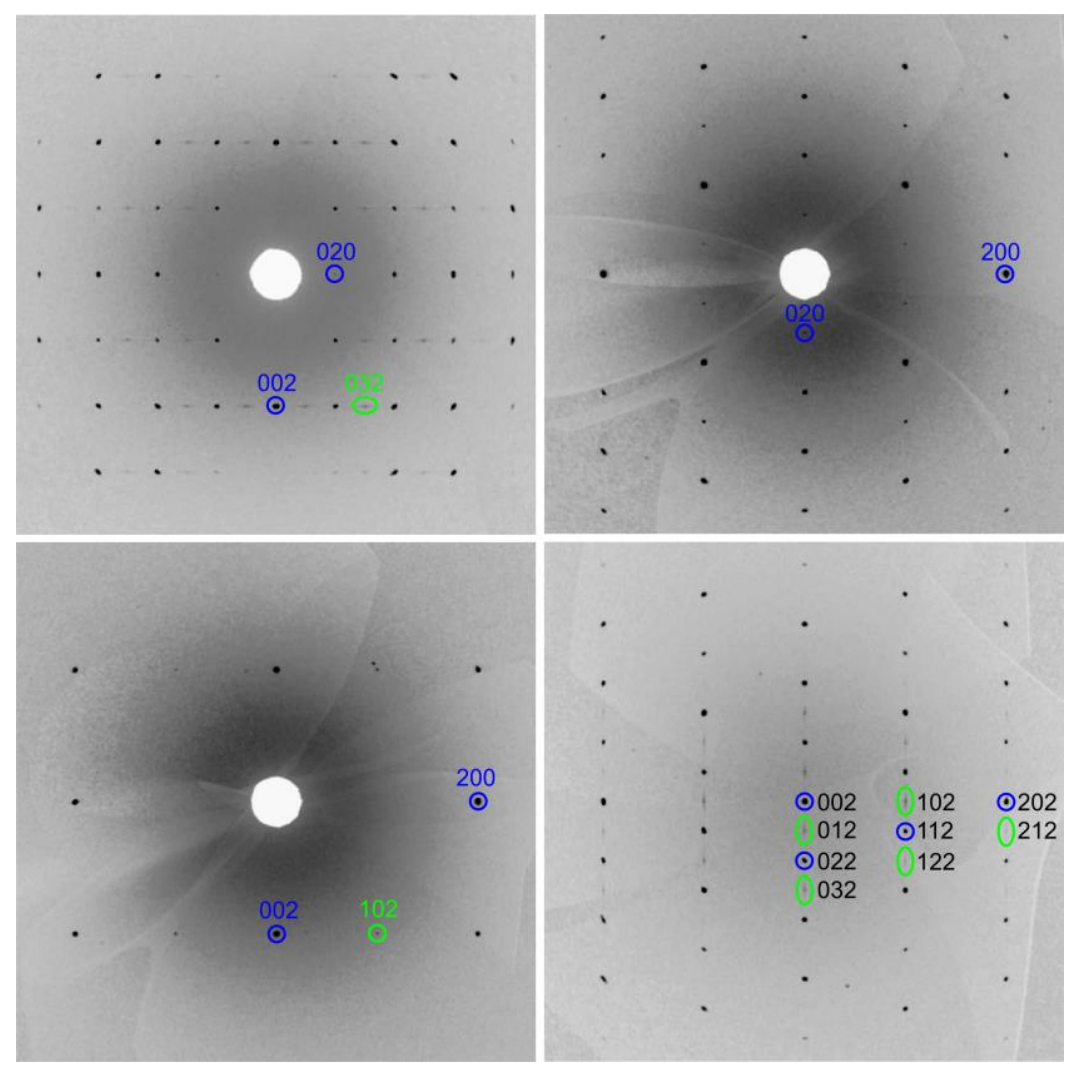

Figure 7 Simulated precession images of the (a) $0 k l$, (b) $h k 0$, (c) $h 0 l$, and (d) $h k 2$ planes from a single crystal of $\mathrm{CaFeSeO}$ obtained on I19 at Diamond. The reflections labelled by blue circles are symmetry allowed for both the $\mathrm{Cmc2}_{1}$ and $\mathrm{Pmcn}$ modifications, but those marked by green circles and ovals are only allowed in the Pmon space group.

Complementary HAADF-STEM and ABF-STEM images in Figure 8 demonstrate that the sense of the tetrahedra tilting distortion can not only change between the adjacent FeSeO layers, but also within the same layer. This results in the splitting of the projected Fe columns into two, which can be distinguished in layers 2-5 and is even more pronounced in layers 12,14 and 16. Following the images in Figure 8 layer-by-layer one can identify that the structure fragments from layer 6 to layer 11 exhibit an ordered arrangement of the Fe positions characteristic for the Pmon polymorph. Starting from layer 12, the Fe positions in every second FeSeO layer are split in two along the $c$ direction, corresponding to a superposition of both polymorphs along the [100] direction, which is schematically illustrated with the structure diagrams at the bottom of the figure. The systematic splitting of the projected Fe positions might indicate that the two polymorphs are separated by planar interfaces parallel to the (100) planes or inclined interfaces, which have some component parallel to (100) planes. It is worth noting that the switching of the character of tilting distortions along the $c$ direction is highly unlikely, because that should introduce considerable strain in the quasi 
1D chains of the $\mathrm{FeSe}_{2} \mathrm{O}_{2}$ tetrahedra. The ED and STEM data highlight that although the two-phase model provides a very good description of the powder diffraction data, there is additional complexity in the system arising from the intergrowth of the two polymorphs.
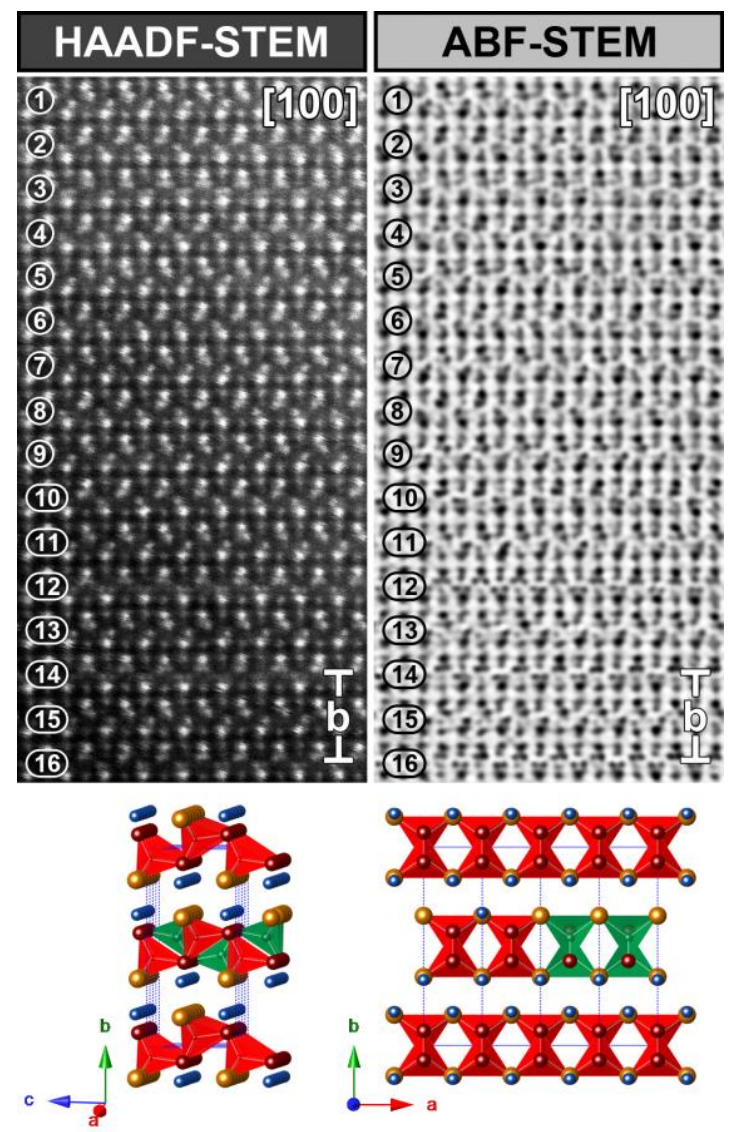

Figure 8 Complementary HAADF-STEM and ABF-STEM images from a region of a crystal with switching of the sense of the $\mathrm{FeSe}_{2} \mathrm{O}_{2}$ tetrahedra tilting in the FeSeO layers (layers 3, 12, 14, 16). Schematic structure diagrams at the bottom depict how the images may be interpreted as disorder extending through the FeSeO blocks along the $a$ axis.

Re-annealing of Sample 2 of $\mathrm{CaFeSeO}$ for 28 days at $740{ }^{\circ} \mathrm{C}$ then furnace cooling did not affect the $C m c 2_{1}$ and $P m c n$ phase ratio ouside the uncertainty of the powder X-ray diffraction measurement (supporting information, Figure S4). As noted in the experimental section, the synthesis of CaFeSeO without substantial impurity phases is restricted to a narrow temperature range, and in this range the two polymorphs are apparently in equilibrium. The work by Han et al., ${ }^{11}$ describes how the minority Pmon polymorph that we describe may be synthesised as small red crystals from a melt of composition " $\mathrm{CaFe}_{2} \mathrm{Se}_{2} \mathrm{O}$ " cooled from $1000{ }^{\circ} \mathrm{C}$ to $700{ }^{\circ} \mathrm{C}$, and it may be that under such conditions the nucleation of this phase is favoured. We have not so far been able to isolate the $C m c 2_{1}$ modification completely phase pure. 
The $\mathrm{FeSe}_{2} \mathrm{O}_{2}$ distorted tetrahedral coordination environment in $\mathrm{CaFeSeO}$ is not reported for any other phases. The bond distances compare well to the most similar $\mathrm{FeSe}_{3} \mathrm{O}$ and $\mathrm{FeS}_{3} \mathrm{O}$ distorted tetrahedral coordination environments given in Table 4. Four coordination for $\mathrm{Fe}^{2+}$ ions is relatively uncommon in oxyselenides; several others adopt $\mathrm{FeO}_{2} \mathrm{Se}_{4}$ distorted octahedral environments: e.g. $\mathrm{La}_{2} \mathrm{O}_{3} \mathrm{Fe}_{2} \mathrm{Se}_{2},{ }^{18} \mathrm{Na}_{2} \mathrm{Fe}_{2} \mathrm{Se}_{2} \mathrm{O},{ }^{19} \mathrm{Ba}_{2} \mathrm{~F}_{2} \mathrm{Fe}_{2} \mathrm{OSe}_{2},{ }^{20}$ and $\beta-\mathrm{La}_{2} \mathrm{FeO}_{2} \mathrm{Se}_{2} .{ }^{21}$

Table 4 Comparison of Fe-O and Fe-Ch bond distances reported here and for similar systems in the literature.

\begin{tabular}{|l|l|l|l|}
\hline Material & Fe-O distance $(\AA)$ & Fe-Ch distance $(\AA)$ & ref \\
\hline $\begin{array}{l}\mathrm{CaFeSeO}: \\
\mathrm{Cmc2}{ }_{1} / \text { Pmcn }\end{array}$ & $\begin{array}{l}1.870(4) / 1.92(2)[1]^{a} \\
1.959(3) / 2.01(1)[1]\end{array}$ & $2.585(2) / 2.53(1)[2]$ & $\begin{array}{l}\text { This work: PND of } \\
\text { sample 3 }\end{array}$ \\
\hline $\mathrm{CaFeSeO}(P m c n)$ & $1.888(8)[1]$ & $2.578(2)[2]$ & 11 \\
& $1.945(8)[1]$ & & \\
\hline $\mathrm{SrFe}_{2} \mathrm{Se}_{2} \mathrm{O}$ & $1.880(2)[1]$ & $\begin{array}{l}2.5305(1)[2] \\
2.4674(7)[1]\end{array}$ & 22 \\
\hline $\mathrm{BaFe}_{2} \mathrm{Se}_{2} \mathrm{O}$ & $1.832(4)[1]$ & $\begin{array}{l}2.560(2)[2] \\
2.486(2)[1]\end{array}$ & 23 \\
\hline $\mathrm{CaFeSO}$ & $1.88(1)[1]$ & $2.391(6)[3]$ (sulfur) & 8,9 \\
\hline
\end{tabular}

${ }^{a}$ numbers in square brackets indicate the number of bonds of a particular type.

\section{Magnetometry}

CaFeSeO shows a sharp transition in the magnetic susceptibility below 159(1) K (Figure 9). The magnetisation does not saturate at $5 \mathrm{~T}$, but the largest value reached of about $0.08 \mu_{\mathrm{B}}$ per formula unit (Figure $9(b)$ ) is consistent with antiferromagnetic ordering with a small canted moment. In addition, strong magnetic hysteresis effects are observed at temperatures below the magnetic ordering transition (bottom left of Figure 9(b)). The magnetisation isotherms obtained after field cooling become open and strongly displaced from the origin at lower temperatures pointing to a glassy component of the magnetism arising from disorder and frustration. The susceptibilities of ferromagnets and spin-glasses have characteristic behaviour in an AC field when measured through the transition as a function of frequency. The sharp magnetic transition temperature at 159(1) K in the AC susceptibility of CaFeSeO (Figure 9 (c)-(d)) shows no frequency dependency, but does have a non-zero imaginary component (Figure $9(d)$ ) pointing to a weak ferromagnetic component. Immediately below the sharp transition at 159(1) K there is a second feature in the AC susceptibility extending from 140 to $158 \mathrm{~K}$, which does have a frequency dependence. The ferromagnetic signature must arise from the long range magnetic ordering of spins in one or both of the phases, and is presumably associated with the formation of a canted antiferromagnetic state. The precise origin of the frequency-dependent feature is unclear, but may originate from the result of the boundary interfaces between the two modifications. 

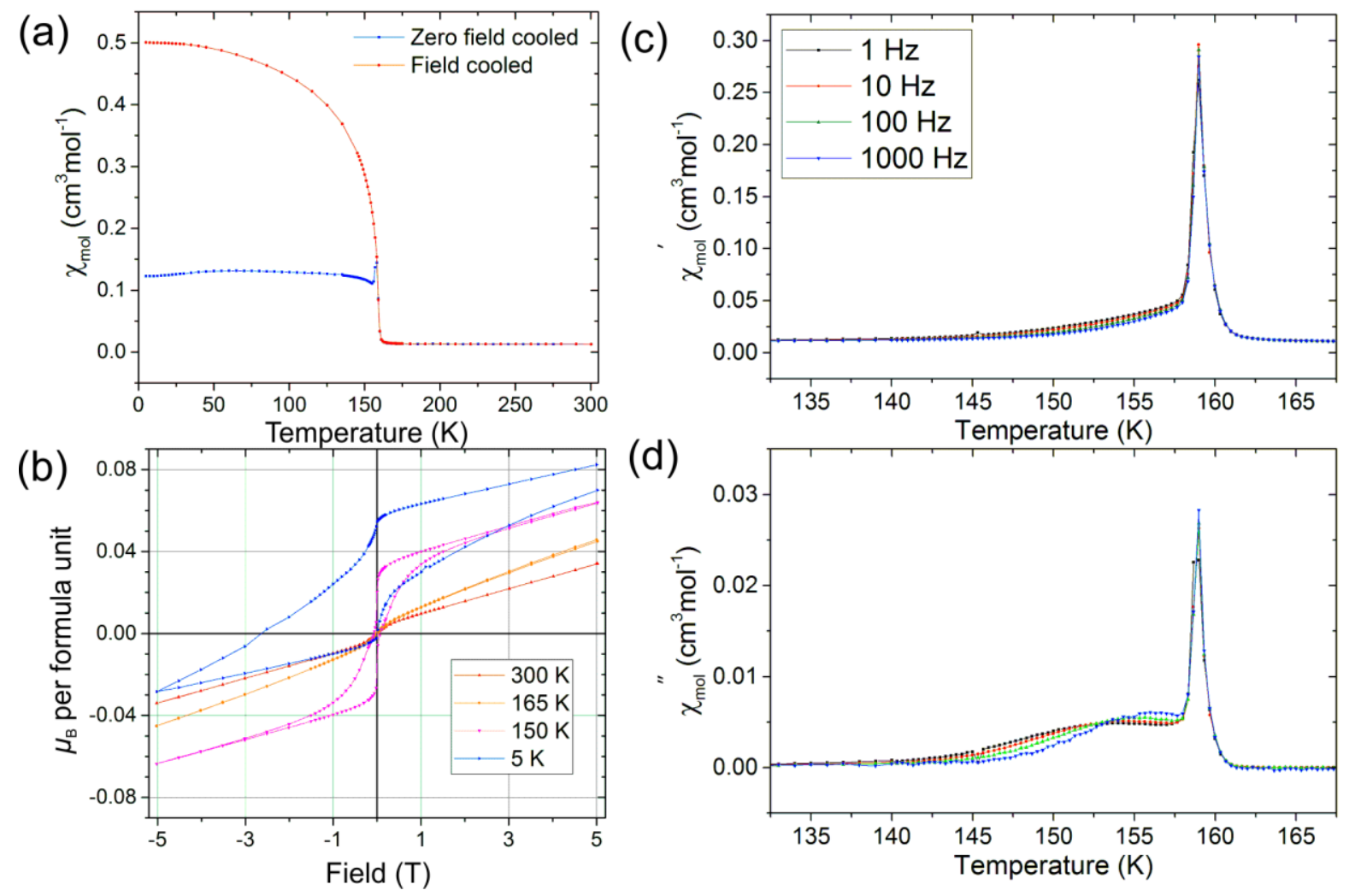

Figure 9 Magnetic susceptibility measurements for Sample 1 of CaFeSeO. (a) magnetic susceptibility as a function of temperature measured under Zero-Field-Cooled (ZFC) and Field-Cooled (FC) conditions with a $50 \mathrm{mT}$ DC field. (b) magnetisation isotherms hysteresis loops $\left(-5 \leq \mu_{0} \mathrm{H} / \mathrm{T} \quad \mathrm{DC} \leq 5\right)$ at room temperature, just above and just below the $159 \mathrm{~K}$ magnetic transition, and at $5 \mathrm{~K}$. (c) real and (d) imaginary components of the magnetic susceptibility of CaFeSeO measured in an AC field with 4 frequencies over the transition.

\section{Magnetic structure}

The neutron diffraction pattern of $\mathrm{CaFeSeO}$ measured on the WISH instrument revealed numerous changes to the intensities of the Bragg peaks below the magnetic transition at 159(1) K. No new peaks appeared suggesting that the magnetic unit cell had the same dimensions as the nuclear cell. Possible orderings of the magnetic moments were tested using the ISODISTORT software. ${ }^{24}$ Refinement of the magnetic structure (Figure 10(a)) revealed that most of the magnetic intensity was accounted for by models for both phases in which $\sim 3.4 \mu_{\mathrm{B}}$ moments localised on the $\mathrm{Fe}^{2+}$ centres (constrained to be equal for both phases) were oriented to point along the $c$ axis (the direction of the $\mathrm{Fe}-\mathrm{O}-\mathrm{Fe}$ chains within the FeSeO layers). Along this $c$ direction nearest-neighbour moments were aligned antiferromagnetically, and along the $a$ direction (the direction of the Fe-Se-Fe chains within the FeSeO layers) the alignment was ferromagnetic. Along the $b$ direction (the stacking direction for the $\mathrm{FeSeO}$ layers), the best agreement with the data was obtained with the $\mathrm{Fe}^{2+}$ moments in both the $\mathrm{Cmc2}_{1}$ phase and the Pmcn phase aligned parallel. The magnetometry data 
indicated a small ferromagnetic canting of the spins; a canted moment along the $a$ direction refined to a value of 0 , while a small canted moment of $0.62(6) \mu_{\mathrm{B}}$ was refined along the $b$ axis perpendicular to the layers. A graphical representation of the combinations of magnetic structures that were trialled is given in Figure $\mathrm{S} 5$ in the supporting information. The final model for the magnetic structure constrained the moments on $\mathrm{Fe}^{2+}$ in the two phases to have not only the same value, but also to be parallel or antiparallel to a single direction in the $\mathrm{FeSe}_{2} \mathrm{O}_{2}$ tetrahedron. This means that the $\mathrm{FeSeO}$ layers all carry a small canting, and in the $C m c 2_{1}$ phase, with FeSeO layers related by the $C$-centring translation, there is a net canting in the crystal, while in the Pmcn phase, with FeSeO layers related by inversion, the cantings in adjacent layers are equal and opposite. The final model places the magnetic structures of the $C m c 2_{1}$ and $P m c n$ phases in Shubnikov groups $C m^{\prime} c 2_{1}{ }^{\prime}$ (space group 36.174 in the Belov, Neronova \& Smirnova (BNS) scheme ${ }^{25}$ ) and $P m^{\prime} c n$ (BNS space group 62.444), respectively as depicted in Figure 9(b). A Rietveld fit against all five detector banks on WISH was performed (Figure S6); the fit to one detector bank is shown in Figure 10(a) and the refined parameters are given in Tables 5-6. Han et al. ${ }^{11}$ characterised the crystal structure of the Pmcn polymorph and used Local Spin Density Approximation (LSDA) and LSDA+U calculations to predict the lowest energy magnetic structure. Their lowest energy magnetically ordered state is indeed the one that we find experimentally for this polymorph.

The local $\mathrm{FeSe}_{2} \mathrm{O}_{2}$ environment is highly distorted from tetrahedral. In both polymorphs the local symmetry at $\mathrm{Fe}$ is $m$ with the two unequal $\mathrm{Fe}-\mathrm{O}$ distances lying within the mirror plane. Thus all degeneracies in the $3 d$ manifold are lifted. $\mathrm{Fe}^{2+}$ has $63 d$ electrons so the least antibonding $3 d$ orbital will be doubly occupied and the other four orbitals will be half occupied. The experimental observation is that the superexchange along the pathway Fe-Se-Fe is ferromagnetic, and since the $\mathrm{Fe}-\mathrm{Se}-\mathrm{Fe}$ bond angle is about $100^{\circ}$ this is consistent with the Goodenough-Kanamori rules when considering $\sigma$-type superexchange which is expected to dominate the $\mathrm{Fe}-\mathrm{Se}-\mathrm{Fe}$ superexchange path. The Fe-O-Fe bond angle is only slightly larger at about $112^{\circ}$ and would be compatible also with ferromagnetic superexchange via $\sigma$-interactions. However in this case, with stronger $\pi$-type covalent interactions between $\mathrm{Fe}$ and $\mathrm{O}$ than between $\mathrm{Fe}$ and $\mathrm{Se}$, there will be several competing exchange pathways; the net result observed experimentally is that there is antiferromagnetic coupling along the Fe-O-Fe pathway. 


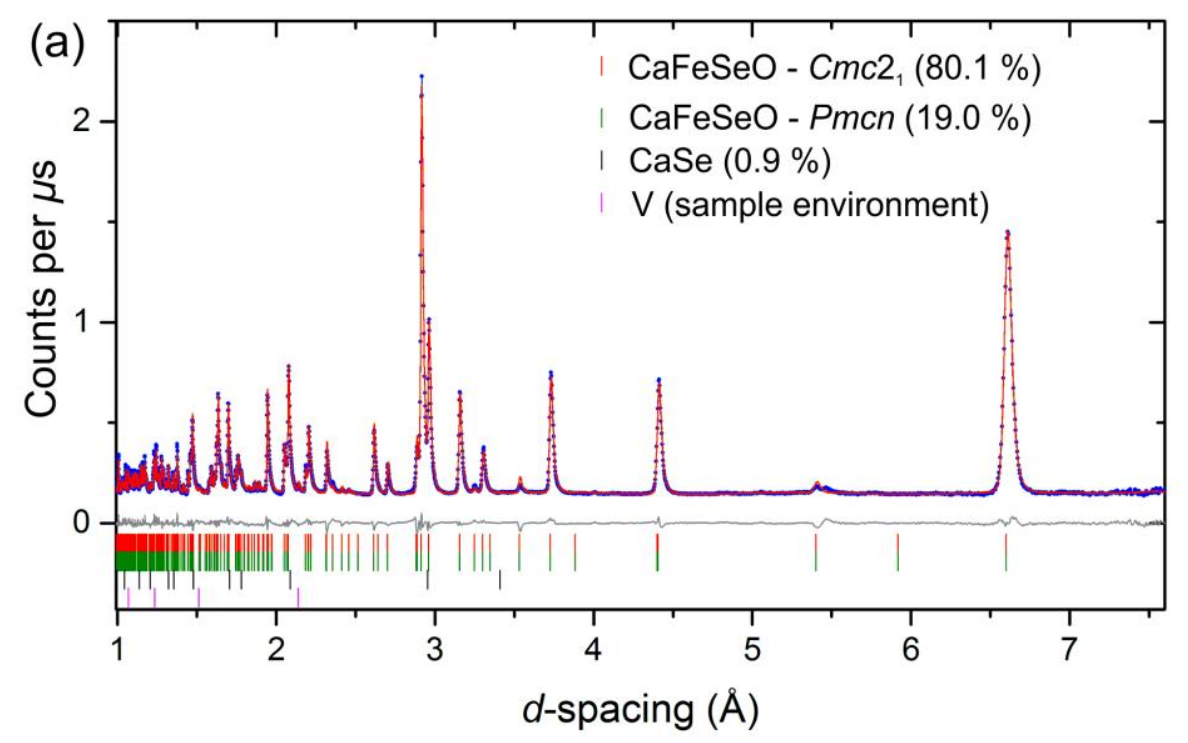

(b)

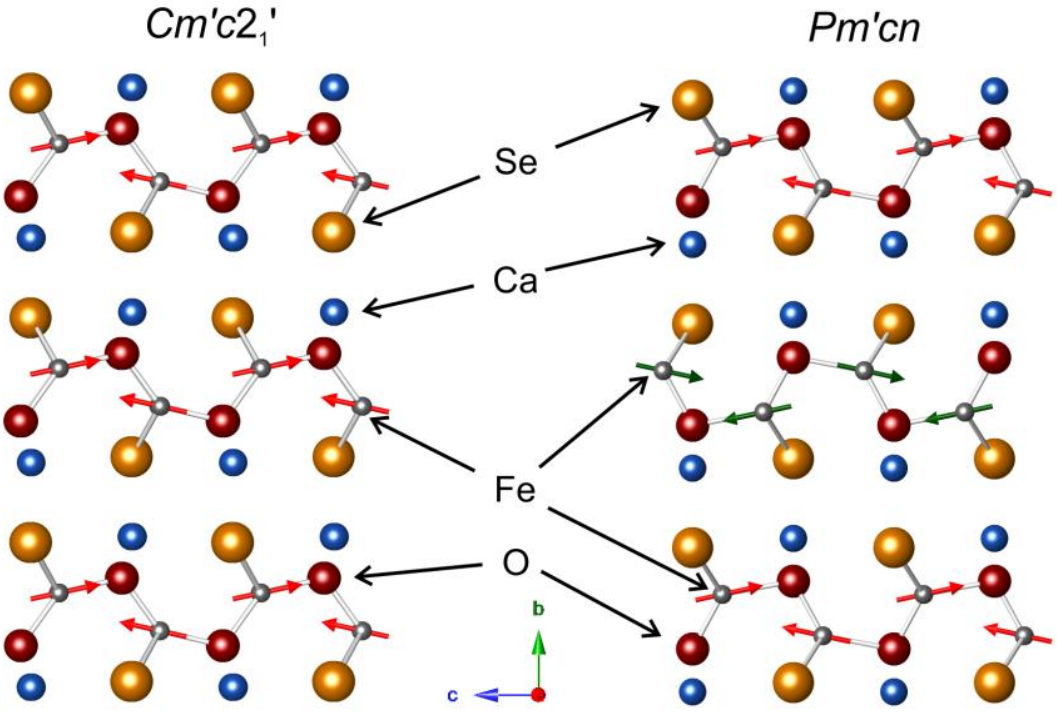

Figure 10 (a) Rietveld refinement against neutron diffraction data at $5 \mathrm{~K}$, showing the fit against WISH bank $3 / 8$ using two CaFeSeO phases (see also Figures S5 and S6). (b) Diagrams showing the magnetic structure of the two phases. The $\mathrm{Fe}^{2+}$ moments were constrained to be equal for the two phases and the orientations of the moments relative to the $\mathrm{FeSe}_{2} \mathrm{O}_{2}$ tetrahedra were also constrained to be similar in the two phases. Data (Blue), fit (red) and difference (grey) are shown. Tick marks indicate reflection positions for the phases present. 
Table 5 Results from a 2 phase Rietveld refinement against PND data of Sample 1 of CaFeSeO at $5 \mathrm{~K}$. Lattice parameters for the two phases were constrained to be equal, due to their minute difference, as shown by high resolution PXRD in Table 1, being beyond the resolution of the WISH instrument.

\begin{tabular}{|c|c|c|}
\hline formula & \multicolumn{2}{|c|}{ CaFeSeO } \\
\hline instrument & \multicolumn{2}{|c|}{ WISH banks $1-10$} \\
\hline temperature & \multicolumn{2}{|c|}{$5 \mathrm{~K}$} \\
\hline crystal system & \multicolumn{2}{|c|}{ orthorhombic } \\
\hline space group & $C m^{\prime} c 2_{1}^{\prime}(\mathrm{BNS} 36.174)$ & $P m^{\prime} \mathrm{cn}$ (BNS 62.444) \\
\hline$a(\AA)$ & \multicolumn{2}{|c|}{$3.88778(7)$} \\
\hline$b(\AA)$ & \multicolumn{2}{|c|}{$13.2103(3)$} \\
\hline$c(\AA)$ & \multicolumn{2}{|c|}{$5.9228(1)$} \\
\hline$V\left(\AA^{3}\right)$ & \multicolumn{2}{|c|}{$304.19(1)$} \\
\hline$\rho\left(\mathrm{g} \mathrm{cm}^{-3}\right)$ & \multicolumn{2}{|c|}{$4.2737(2)$} \\
\hline$R_{w p}(\%)$ & \multicolumn{2}{|c|}{3.41} \\
\hline polymorph fraction (\%) & $81(1)$ & 19(1) \\
\hline $\mathrm{Fe}-\mathrm{O}(\AA)$ & $\begin{array}{l}1.875(3) \\
1.972(3)\end{array}$ & $\begin{array}{l}1.81(1) \\
1.996(9)\end{array}$ \\
\hline Fe-Se $(\AA ̊)[2]$ & $2.594(2)$ & $2.557(5)$ \\
\hline $\mathrm{Ca}-\mathrm{O}(\AA \AA)[2]$ & $2.316(3)$ & $2.298(9)$ \\
\hline Ca-Se $(\AA ̊)[1]$ & $2.918(3)$ & $2.865(9)$ \\
\hline [1] & $3.012(3)$ & $3.081(9)$ \\
\hline [2] & $3.013(3)$ & $3.22(1)$ \\
\hline $\mathrm{Fe}-\mathrm{O}-\mathrm{Fe}\left({ }^{\circ}\right)$ & $111.1(1)$ & $113.0(5)$ \\
\hline $\mathrm{O}-\mathrm{Fe}-\mathrm{O}\left({ }^{\circ}\right)$ & $136.0(1)$ & $134.9(4)$ \\
\hline $\mathrm{Fe}-\mathrm{Se}-\mathrm{Fe}\left({ }^{\circ}\right) / \mathrm{Se}-\mathrm{Fe}-\mathrm{Se}\left({ }^{\circ}\right)$ & $97.08(8)$ & 98.9(3) \\
\hline
\end{tabular}

Table 6 Structural coordinates for the $C m^{\prime} c 2_{1}{ }^{\prime}$ phase (BNS scheme: 36.174 and the $P m^{\prime} c n$ phase (BNS scheme: 62.444 at $5 \mathrm{~K}$ measured on WISH.

$\mathrm{Cm}^{\prime} \mathbf{c 2}^{\prime}$

\begin{tabular}{|l|l|l|l|l|l|}
\hline atom & site & $x$ & $y$ & $z$ & $U_{\text {iso }}\left(\AA^{2}\right)$ \\
\hline $\mathrm{Ca}$ & $4 a$ & 0 & $0.3317(3)$ & $0.0079(4)$ & $0.0005(5)$ \\
\hline $\mathrm{Fe}^{a}$ & $4 a$ & 0.5 & $0.0430(1)$ & $0.1372(3)$ & $0.0049(3)$ \\
\hline $\mathrm{Se}$ & $4 a$ & 0 & $0.6575(1)$ & 0 & $0.0001(4)$ \\
\hline $\mathrm{O}$ & $4 a$ & 0.5 & $0.0753(2)$ & $0.4623(4)$ & $0.0028(4)$ \\
\hline
\end{tabular}

\section{Pm'cn}

\begin{tabular}{|l|l|l|l|l|l|}
\hline $\mathrm{Ca}^{b}$ & $4 c$ & 0.25 & $0.587(2)$ & $0.738(1)$ & $0.0005(5)$ \\
\hline $\mathrm{Fe}^{a}$ & $4 c$ & 0.25 & $0.2074(4)$ & $0.1120(8)$ & $0.0049(3)$ \\
\hline $\mathrm{Se}$ & $4 c$ & 0.25 & $0.6068(5)$ & $0.2197(8)$ & $0.0001(4)$ \\
\hline $\mathrm{O}$ & $4 c$ & 0.25 & $0.3212(8)$ & $0.283(1)$ & $0.0028(4)$ \\
\hline
\end{tabular}

${ }^{a}$ In both structures $\mathrm{Fe}^{2+}$ ions carry a localised moment of 3.52(1) $\mu_{\mathrm{B}}\left(\boldsymbol{M}_{\mathbf{x}}=0 \mu_{\mathrm{B}} ; \boldsymbol{M}_{\boldsymbol{y}}=-0.62(6) \mu_{\mathrm{B}} ; \boldsymbol{M}_{\mathbf{z}}=\right.$ 3.46(1) $\mu_{\mathrm{B}}$ (Figure 10(b))).

${ }^{b}$ The coordinates of $\mathrm{Ca}$ in the minority Pmcn phase have been restrained to within $0.02 \AA$ difference from those of sample 3 in Table 2, which have been more accurately determined by Rietveld refinement against GEM and POLARIS neutron diffraction data. 


\section{Exchange-striction}

The evolution of the $\mathrm{CaFeSeO}$ nuclear crystal structure when cooled through its magnetic transition at 159(1) K was probed using synchrotron PXRD (Figure 11). High signal-to-noise ratio diffraction patterns collected at room temperature and $110 \mathrm{~K}$ showed no changes in peak intensity or appearance aside from that occurring from thermal contraction, suggesting that the nuclear symmetry is not broken through the magnetic transition. The ratio of $C m c 2_{1}$ and $P m c n$ phases used at ambient temperatures continues to model the low temperature data well.

Systematic Rietveld refinement was carried out against 144 diffraction patterns gathered at $1.3 \mathrm{~K}$ intervals on cooling using the two phase model. A discontinuity in the thermal contraction of the three lattice parameters is evident at the temperature of the magnetic transition (159(1) K) and we ascribe this to an exchange-striction effect, often found for antiferromagnetic compounds. ${ }^{26-28}$ The lattice parameters $a$ and $c$, which describe the plane parallel to the puckered FeSeO layers, expand, while the lattice parameter $b$, perpendicular to these layers, contracts. The unit cell volume is unperturbed by the magnetic transition (Supporting Information, Figure S7). The structural transition is small and only evident in the lattice parameters which are determined with a much greater precision than bond lengths and angles.
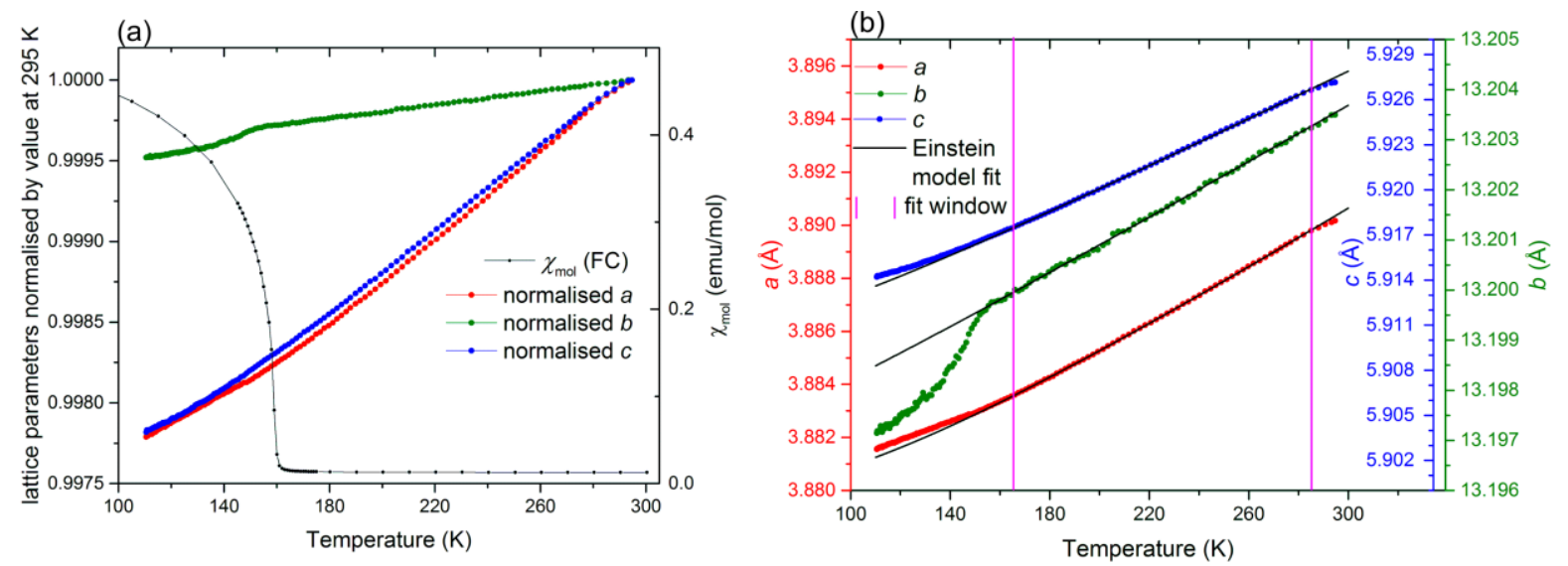

Figure 11 (a) Refined lattice parameters of $\mathrm{CaFeSeO}$ as a function of temperature, normalised to lattice parameter at room temperature. A discontinuity occurs in each parameter's contraction, coinciding with the transition in the magnetic susceptibility (data from Figure 8(a) also shown). (b) fits to the thermal contraction of each lattice parameter based on Eqn 1.

No differences in the temperature dependence of the lattice parameters of the two phases were evident when using a two phase Rietveld model. So in order to determine the thermal contraction coefficients and size of the exchange-striction, a Pawley (model-independent) refinement of a single 
set of orthorhombic unit cell parameters was performed in the space group $P m c 2_{1}$ - a subgroup of both $C m c 2_{1}$ and Pmcn; a Stephens type anisotropic peak broadening function ${ }^{29}$ was applied to better model the peak shapes. The refined lattice parameters are plotted in Figure 11(b) where the thermal contraction of $\mathrm{CaFeSeO}$ has been fitted using an Einstein-type dependence (Eqn 1) over the range 165-285 K:

$$
x=x_{0}+\frac{C_{\mathrm{E}}}{\exp \left({ }_{\mathrm{E}} / T^{-1}\right)}
$$

where $x$ is the lattice parameter $a, b$ or $c, x_{0}$ is expected lattice parameter at $0 \mathrm{~K}, C_{\mathrm{E}}$ is the Einstein constant and $\theta_{\mathrm{E}}$ is the Einstein temperature. The coefficients obtained from these fits are given in Table 7.

Each of the lattice parameters is found to deviate from Eqn 1 at temperatures below the magnetic transition at 159(1) K. The coefficients describing the exchange-striction for the three axes $\lambda_{a}, \lambda_{b}$, and $\lambda_{c}$, at a particular temperature are defined in Eqn 2:

$$
\lambda_{a}=\frac{\Delta a}{a}, \lambda_{b}=\frac{\Delta b}{b}, \lambda_{c}=\frac{\Delta c}{c}
$$

Where $\Delta a$ is the difference between the measured value of $a$ at a particular temperature and the expected value of $a$ at the same temperature based on the extrapolation of the fit to Eqn 1 below the magnetic transition temperature. The values of the $\lambda$ coefficients at $110 \mathrm{~K}$ expressed in parts per million (ppm) are given in Table 7.

Table 7. Parameters used to fit the thermal contraction of the lattice parameters above the magnetic transition according to Eqn 1 (fits shown in Figure 11(b)) and the magnetostriction coefficients $(\lambda)$ (Eqn 2).

\begin{tabular}{|l|l|l|l|l|}
\hline $\begin{array}{l}\text { unit cell } \\
\text { parameter }\end{array}$ & $\begin{array}{l}\text { parameter zero } \\
\text { value, } x_{0}(\AA)\end{array}$ & $C_{\mathrm{E}}(\AA)$ & $\theta_{\mathrm{E}}(\mathrm{K})$ & $\lambda(\mathrm{ppm})$ \\
\hline$a$ & $3.8799(7)$ & $0.0174(5)$ & $289(6)$ & +140.5 \\
\hline$b$ & $13.1968(4)$ & $0.04(2)$ & $127(73)$ & -99.8 \\
\hline$c$ & $5.9102(2)$ & $0.016(1)$ & $190(11)$ & +119 \\
\hline
\end{tabular}

Deviation of the lattice parameters from the Einstein-type dependence below the onset of long range magnetic order can be rationalised by considering the sign of the super-exchange interactions within the FeSeO puckered layers and the orientation of the magnetic moments. 
Ferromagnetic alignment of moments along the $a$ direction via the Fe-Se-Fe coupling results in a positive deviation from the normal thermal contraction effects of the lattice parameter $a$. Figure 11(a) shows that this deviation commences at $T_{\mathrm{N}}$. Along the $c$ direction via the $\mathrm{Fe}-\mathrm{O}-\mathrm{Fe}$ antiferromagnetic coupling, a slight negative deviation of the lattice parameter from the Einstein dependence (i.e. an enhanced contraction) would be expected. However this will be opposed by the weak ferromagnetic component of this interaction associated with the spin canting. Indeed the behaviour of the $c$ lattice parameter shows a net positive deviation from the Einstein behaviour which becomes apparent about $20 \mathrm{~K}$ below $T_{\mathrm{N}}$ and coincides with the temperatures at which the canted moment approaches saturation. We propose that the contraction of the $b$ axis perpendicular to the layers is a response to the net expansion within the layers such that the cell volume is preserved.

\section{Conclusions}

We have shown that the antiferromagnet $\mathrm{CaFeSeO}$ can be synthesised stoichiometric over a very narrow temperature range which accounts for why other investigations in this phase field have either not succeeded in isolating the phase ${ }^{4}$ or have synthesised $\mathrm{CaFeSeO}$ as a minority phase along with phases of other compositions. ${ }^{11}$ The compound is dimorphic, presumably because the two crystallographic forms have similar energies, and the two polymorphs could not be isolated in stoichiometric samples. The crystal structures of both polymorphs are related to the structure of BaZnSO and BaCoSO by a tilting of the tetrahedra within puckered two-dimensional FeSeO layers composed of $\mathrm{FeSe}_{2} \mathrm{O}_{2}$ tetrahedra which share all their vertexes. The detailed analysis of transmission electron microscopy data, together with bulk single crystal and powder X-ray, and neutron powder diffraction measurements show that the two polymorphs differ in the relative orientations of the tilting distortion. In one polymorph with $C m c 2_{1}$ symmetry, the FeSeO layers are related by the $C$ centring translation, while in the second polymorph, with Pmcn symmetry, ${ }^{11}$ the layers are related by inversion and the structure is similar to that adopted for $\mathrm{SrZnO}_{2}$. The Bragg scattering resulting from magnetic order of the $\mathrm{Fe}^{2+}$ moments can also be interpreted using the two-phase model with a small canting of the moments within each FeSeO layer resulting in a weak ferromagnetism in the $\mathrm{Cmc2}_{1}$ phase which is evident in magnetometry measurements. According to TEM investigations, both polymorphs can randomly intergrow in different regions of individual powder grains, and the resulting interfaces likely account for the glassiness which is also evident in the magnetometry and 
gives rise to displaced and open DC magnetisation isotherms and a frequency dependent contribution to the AC susceptibility.

\section{Associated Content.}

\section{Supporting Information}

The Supporting Information is available free of charge on the ACS Publications website at DOI: XXXXX Rietveld refinements against all available banks of data, tables of refinement results, additional TEM images (PDF).

\section{Author Information.}

\section{Corresponding Author}

*email: simon.clarke@chem.ox.ac.uk

\section{Acknowledgements.}

We acknowledge the financial support of the EPSRC (Grants EP/I017844/1 and EP/M020517/1), the Leverhulme Trust (RPG-2014-221) and the Diamond Light Source (studentship support for S. J. Cassidy). We thank the ESTEEM2 network for enabling the electron microscopy investigations and the ISIS facility and the Diamond Light Source Ltd for the award of beam time. We thank Dr P. Manuel for assistance on WISH, Dr R. I. Smith for assistance on GEM and POLARIS and Dr C. Murray and $\operatorname{Dr}$ A. Baker for assistance on 111. 


\section{References}

(1) Clarke, S. J.; Adamson, P.; Herkelrath, S. J. C.; Rutt, O. J.; Parker, D. R.; Pitcher, M. J.; Smura, C. F. Structures, Physical Properties, and Chemistry of Layered Oxychalcogenides and Oxypnictides. Inorg. Chem. 2008, 47, 8473-8486.

(2) Ueda, K.; Inoue, S.; Hirose, S.; Kawazoe, H.; Hosono, H. Transparent p-type semiconductor: LaCuOS layered oxysulfide. Appl. Phys. Lett. 2000, 77, 2701-2703.

(3) Kamihara, Y.; Watanabe, T.; Hirano, M.; Hosono, H. Iron-Based Layered Superconductor $\mathrm{La}\left[\mathrm{O}_{1-x} \mathrm{~F}_{x}\right] \mathrm{FeAs}(x=0.05-0.12)$ with $T_{\mathrm{c}}=26 \mathrm{~K} . \mathrm{J}$. Am. Chem. Soc. 2008, 130, 3296-3297.

(4) Hosono, H.; Tanabe, K.; Takayama-Muromachi, E.; Kageyama, H.; Yamanaka, S.; Kumakura, H.; Nohara, M.; Hiramatsu, H.; Fujitsu, S. Exploration of new superconductors and functional materials, and fabrication of superconducting tapes and wires of iron pnictides. Sci. Technol. Adv. Mater. 2015, 16, 033503.

(5) Broadley, S.; Gál, Z. A.; Corà, F.; Smura, C. F.; Clarke, S. J. Vertex-linked $\mathrm{ZnO}_{2} \mathrm{~S}_{2}$ tetrahedra in the oxysulfide BaZnOS: a new coordination environment for zinc in a condensed solid. Inorg. Chem. 2005, 44, 9092-9096.

(6) Valldor, M.; Rössler, U. K.; Prots, Y.; Kuo, C. Y.; Chiang, J. C.; Hu, Z. W.; Pi, T. W.; Kniep, R.; Tjeng, L. H. Synthesis and Characterization of $\mathrm{Ba}[\mathrm{CoSO}]$ : Magnetic Complexity in the Presence of Chalcogen Ordering. Chem. Eur. J. 2015, 21, 10821-10828.

(7) Sambrook, T.; Smura, C. F.; Clarke, S. J.; Ok, K. M.; Halasyamani, P. S. Structure and Physical Properties of the Polar Oxysulfide CaZnOS. Inorg. Chem. 2007, 46, 2571-2574.

(8) Salter, E. J. T.; Blandy, J. N.; Clarke, S. J. Crystal and Magnetic Structures of the Oxide Sulfides CaCoSO and BaCoSO. Inorg. Chem., 2016, 55, 1697-1701.

(9) Delacotte, C.; Pérez, O.; Pautrat, A.; Berthebaud, D.; Hébert, S.; Suard, E.; Pelloquin, D.; Maignan, A. Magnetodielectric Effect in Crystals of the Noncentrosymmetric CaOFeS at Low Temperature. Inorg. Chem. 2015, 54, 6560-6565.

(10) Jin, S. F.; Huang, Q.; Lin, Z. P.; Li, Z. L.; Wu, X. Z.; Ying, T. P.; Wang, G.; Chen, X. L. Twodimensional magnetic correlations and partial long-range order in geometrically frustrated CaOFeS with triangle lattice of Fe ions. Phys. Rev. B 2015, 91, 094420.

(11) Han, F.; Wang, D.; Malliakas, C. D.; Sturza, M.; Chung, D. Y.; Wan, X.; Kanatzidis, M. G. (CaO)(FeSe): A Layered Wide-Gap Oxychalcogenide Semiconductor. Chem. Mater. 2015, 27, 5695-5701.

(12) McQueen, T. M.; Huang, Q.; Ksenofontov, V.; Felser, C.; Xu, Q.; Zandbergen, H.; Hor, Y. S.; Allred, J.; Williams, A. J.; Qu, D.; Checkelsky, J.; Ong, N. P.; Cava, R. J. Extreme sensitivity of superconductivity to stoichiometry in Fe $1+\delta$ Se. Phys. Rev. B 2009, 79, 014522.

(13) Thompson, S. P.;. Parker, J. E; Potter. J.; Hill, T. P.; Birt, A.; Cobb, T. M.; Yuan, F.; Tang, C. C. Beamline 111 at Diamond: A new instrument for high resolution powder diffraction. Review of Scientific Instruments 2009, 80, 075107.

(14) Nowell, H.; Barnett, S. A.; Christensen, K. E.; Teat, S. J.; Allan, D. R. I19, the smallmolecule single-crystal diffraction beamline at Diamond Light Source. J. Synchrotron Rad. 2012, 19, 435-441. 
(15) Coelho, A. A. TOPAS Academic, Version 5; Coelho Software: Brisbane, Australia, 2012.

(16) Altomare, A.; Cascarano, G.; Giacovazzo, C.; Guagliardi, A.; Burla, M. C.; Polidori, G.; Camalli, M. SIR92 - a program for automatic solution of crystal structures by direct methods. J. Appl. Crystallogr. 1994, 27, 435-435.

(17) Betteridge, P. W.; Carruthers, J. R.; Cooper, R. I.; Prout, K.; Watkin, D. J. CRYSTALS version 12: software for guided crystal structure analysis. J. Appl. Crystallogr. 2003, 36, 1487-1487.

(18) Mayer, J. M.; Schneemeyer, L. F.; Siegrist, T.; Waszczak, J. V.; Van Dover, B. New Layered Iron-Lanthanum-Oxide-Sulfide and -Selenide Phases: $\mathrm{Fe}_{2} \mathrm{La}_{2} \mathrm{O}_{3} \mathrm{E}_{2}(\mathrm{E}=\mathrm{S}, \mathrm{Se})$. Angew. Chemie Int. Ed. English 1992, 31, 1645-1647.

(19) He, J. B.; Wang, D. M.; Shi, H. L.; Yang, H. X.; Li, J. Q.; Chen, G. F. Synthesis, structure, and magnetic properties of the layered iron oxychalcogenide $\mathrm{Na}_{2} \mathrm{Fe}_{2} \mathrm{Se}_{2} \mathrm{O}$. Phys. Rev. B 2011, 84, 205212.

(20) Kabbour, H.; Janod, E.; Corraze, B.; Danot, M.; Lee, C.; Whangbo, M.-H.; Cario, L. Structure and Magnetic Properties of Oxychalcogenides $\mathrm{A}_{2} \mathrm{~F}_{2} \mathrm{Fe}_{2} \mathrm{OQ}_{2}(\mathrm{~A}=\mathrm{Sr}, \mathrm{Ba} ; \mathrm{Q}=\mathrm{S}$, Se) with $\mathrm{Fe}_{2} \mathrm{O}$ Square Planar Layers Representing an Antiferromagnetic Checkerboard Spin Lattice. J. Am. Chem. Soc. 2008, 130, 8261-8270.

(21) McCabe, E. E.; Free, D. G.; Mendis, B. G.; Higgins, J. S.; Evans, J. S. O. Preparation, Characterization, and Structural Phase Transitions in a New Family of Semiconducting Transition Metal Oxychalcogenides $\beta-\mathrm{La}_{2} \mathrm{O}_{2} \mathrm{MSe}_{2}(\mathrm{M}=\mathrm{Mn}, \mathrm{Fe})$. Chem. Mater. 2010, 22, 61716182.

(22) Huh, S.; Prots, Y.; Adler, P.; Tjeng, L. H.; Valldor, M. Synthesis and Characterization of Frustrated Spin Ladders $\mathrm{SrFe}_{2} \mathrm{~S}_{2} \mathrm{O}$ and $\mathrm{SrFe}_{2} \mathrm{Se}_{2} \mathrm{O}$. Eur J. Inorg. Chem. 2015, 18, 2982-2988.

(23) Han, F.; Wan, X.; Shen, B.; Wen, H.-H. BaFe $\mathrm{Se}_{2} \mathrm{O}$ as an iron-based Mott insulator with antiferromagnetic order. Phys. Rev. B 2012, 86, 014411.

(24) Campbell, B. J.; Stokes, H. T.; Tanner, D. E.; Hatch, D. M. ISODISPLACE: a web-based tool for exploring structural distortions. J. Appl. Crystallogr. 2006, 39, 607-614.

(25) Litvin, D. B. Magnetic Group Tables: 1-, 2- and 3-Dimensional Magnetic Subperiodic Groups and Magnetic Space Groups, International Union of Crystallography 2013; ISBN 9780-9553602-2-0 doi:10.1107/9780955360220001.

(26) Greenwald, S and Smart, J. S. Deformations in the Crystal Structures of Antiferromagnetic Compounds. Nature 1950, 166, 523-524.

(27) Choi, Y. J.; Yi, H. T.; Lee, S.; Huang, Q.; Kiryukhin, V.; Cheong, S.-W. Ferroelectricity in an Ising Chain Magnet. Phys. Rev. Lett. 2008, 100, 047601.

(28) Attfield, J. P. Exchange striction and exchange constants in $\mathrm{Bi}_{2} \mathrm{CuO}_{4}$. J. Phys.: Condens. Matter 1989, 1, 7045-7053.

(29) Stephens, P. Phenomenological model of anisotropic peak broadening in powder diffraction. J.Appl. Crystallogr., 1999, 32, 281-289. 


\section{For Table of Contents Only}

The layered antiferromagnet $\mathrm{CaFeSeO}$ displays complex microstructure resulting from competition between two polymorphs which differ in the relative orientations of their $\mathrm{FeSe}_{2} \mathrm{O}_{2}$ tetrahedra in adjacent layers.

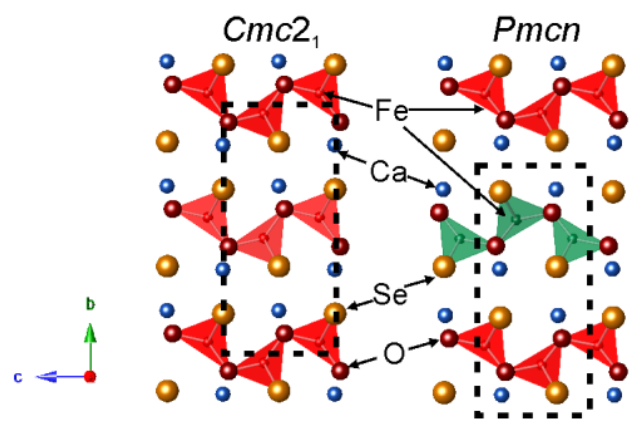

